UC.15

Issued: September 1981

\title{
Perimeter Safeguards Techniques for Uranium-Enrichment Plants
}

by

P. E. Fohlau and W. H. Chambers

Contributors

H. F. Atwater

J. M. Bieri

C. N. Henry

J. T. Caldwell

W. E. Kunz

E. J. Dowdy

N. Nicholson

J. L. Evans

T. E. Sampson

R. D. Hastings

G. M. Worth

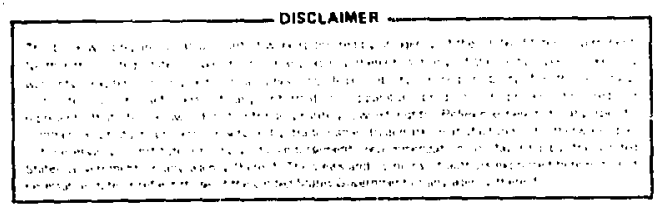

$\Gamma$ 
ABSTRACT

I. INTRODUCTION

II. RADIATION CHARACTERISTICS OF URANIUM HEXAFLUORIDE . 4

A. Gamma Radiation from Uranium Isotopes . . . . . . . . 4

B. Other Features in UF 6 Cylinder Gamma-Ray Spectra . . . . . S

C. Examples of Gamma-Ray Spectra of $\mathrm{UF}_{6}$ Cylinders . . . . . . 5

D. Neutron Output from UF 6 Cylinders. . . . . . . . . 8

III. INSTRUMENTATION FOR PERIMETER SURVEILLANCE . . . . . 9

A. Personnel Doorway Monitor . . . . . . . . . . . . . 9

B. Shipping Dock Monitor .. . . . . . . . . . . . 15

C. Hand-Held Personnel, Package, and Vehicle Monitor . . . . 20

D. Package Monitor . . . . . . . . . . . . . . .21

1. Passive Neutron Detection. . . . . . . . . . . .22

2. Passive Gamma-Ray Detection. . . . . . . . . . . . 22

3. Radiography . . . . . . . . . . . . . . 22

4. Neutron Radiography . . . . . . . . . . . . 23

5. Neutron Transmission . . . . . . . . . . . .23

6. Active Neutron Interrogation Using Delayed Neutrons . . . 23

7. Active Neutron Interrogation Using Prompt

Fission Neutrons . . . . . . . . . . . . 26

IV. PORTABLE INSTRUMENTS FOR VERIFYING

URANIUM ENRICHMENT . . . . . . . . . . . . . . 30

A. Gamma-Ray Enrichment Meter . . . . . . . . . . 30

B. An Alternative Gamma-Ray Method Using $23 \dot{S} \cup$ and

238 U Decay Radiation . . . . . . . . . . . . . 31

C. UF 6 Enrichment Verification with a Thermal Neutron Detector . 33

D. Passive Neutron Measurements with ${ }^{4} \mathrm{He}$ Detectors . . . . . .38

E. Active Neutron Interrogation and ${ }^{4} \mathrm{He}$ Detectors . . . . . .41

F. Radiography of UF 6 Cylinders . . . . . . . . . . 43

G. Summary of the Verification Techniques........45

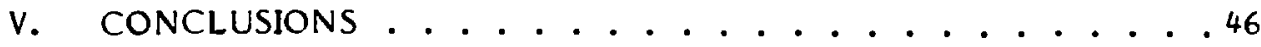

APPENDIX A. STANDARD UF 6 CYLINDER DATA . . . . . . . . .47

APPENDIX B. CALCULATED RESPONSE FOR A

TWO-GAMMA-RAY ENRICHMENT METER. . . . . .5I

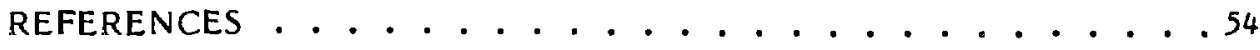

GLOSSARY. . . . . . . . . . . . . . 56 


\title{
PERIMETER SAFEGUARDS TECHNIQUES \\ FOR URANIUM ENRICHMENT PLANTS
}

\author{
by
}

\author{
P. E. Fehlau and W. H. Chambers
}

Contributors

H. F. Atwater, J. M. Bieri, J. T. Caldwell, E. J. Dowdy, J. L. Evans, R. D. Hastings, C. N. Henry, W. E. Kunz, N. Nicholson, T. E. Sampson, and G. M. Worth

\begin{abstract}
In 1972, a working group of the International Atomic Energy Agency identified a goal to develop and evaluate perimeter safeguarc's for uranium isotope anrichment plants. As part of the United States's response to that goal, Los Alamos Detection and Verification personnel studied gamma-ray and neutron emissions from uranium hexafluoride. They developed instruments that use the emissions to verify uranium enrichment and to monitor perimeter personnel and shipping portals. Unattended perimeter monitors and r.and-held verification instruments were evaluated in field measurements and, when possible, were loaned to enrichment facilities for trials. None of the seven package monitoring techniques that were investigated proved entirely satisfactory for an unattended monitor. They either revealed proprietary information about centrifuge design or were subject to interference by shielding materials that could be present in a package. Further evaluation in a centrifuge facility may help in developing an acceptable attended package monitor.
\end{abstract}

\section{INTRODUCTION}

This report describes work, completed in 1975, on perimeter safeguards techniques for uranium enrichment plants. No final report was published and only a synopsis of the entire program appears in the literature. Recently, safeguards for enrichment plants is again of interest with the goal of developing techniques for possible application to a centrifuge plant planned for construction at Portsmouth, Ohio. As a result of the new research 
interest, some duplication of work has taken place. This report summarizes our work up to 1975 and references later applications and evaluations of the techniques and equipment that we discuss.

The International Atomic Energy Agency (IAEA) Working Group meeting on Inspection Procedures at Isotope Enrichment Plants, held in Vienna in June 1972, emphasized the need to develop and evaluate diversion safeguards techniques to be applied at the perimeter of an isotope separation plant. The plant perimeter was located outside the isotope separation cascade area, because IAEA access to the cascade would normally be denied for proprietary or security reasons. After preliminary discussions with personnel from the Department of Energy (DOE) and Arris Control and Disarmament Agency (ACDA), the Los Alamos Detection and Verification Group agreed in August 1972 to generate and test the nuclear Identification, verification, and surveillance instrumentation required to verify material flow across a plant boundary. Testing was planned to analyze the effectiveness of the radiation surveillance components under normal operating conditions, to evaluate their susceptibility to deliberate countermeasures, and to establish their degree of intrusion into proprietary areas. Equipment and manpower costs for operation of the components, as well as the impact of the instruments on normal plant operation, were to be considered in the evaluation of techniques.

In view of the need to consider economics, effectiveness, and acceptability to IAEA inspectors and plant management, alternatives were developed and presented in the course of the study. The conceptual alternatives ranged between a continuous mass and isotopic balance on the one extreme and simple detection of kilogram quantities of highly enriched material crossing the perimeter at vehicular portals on the other. Insofar as possible, without having a specific plant for guidance, choices were made among alternatives, and selected components of the radiation surveillance system were taken completely through the prototype hardware and field testing stage. Where appropriate, tamper-indicating techniques for instrumentation developed by Sandia Laboratories were applied.

Program guidance was provided by a joint DOE/ACDA working group that met periodically. An overall project plan developed in November 1972 specified the following efforts:

a. Identify specific devices, techniques, instruments, and methods for an effective perimeter surveillance system.

b. Outline and describe IAEA inspector activities that are needed for containment and surveillance as well as materials accountability.

c. Define operational characteristics needed to carry out inspector activities in item b.

d. Assess availability of and evaluate potential effectiveness of techniques that are candidates for accomplishing activities in item $b$ and that satisfy the operational characteristics in item c. operation.

e. Develop necessary technology for tamper-resistant, unattended 
f. Conduct field tests to evaluate effectiveness of the techniques when faced with evasive actions and to determine the impact of the techniques on plant operations.

Tasks $a$ and $b$ were completed as part of the project plan. The plan identified activities that used radiation instrumentation to verify enrichment of $U_{6}$ in cylinders and to monitor personnel, vehicles, and packages. Tasks $\mathrm{C}$ thruugh $f$ are the subject of this report.

A 2-yr project was to be completed by June 1974 with prototype demonstrations at a United States (US) gas diffusion plant. We expected international safeguards to be applied first to centrifuge plants, hence for model purposes, we selected the gas centrifuge pilot plants of the Tripartite Group at Almelo and Capenhurst. Model plant information (outside the restricted area) including centrifuge characteristics, material balance areas and plant layouts, personnel and vehicle traffic, material throughput, and container configurations was provided by $H$. Kouts in December 1972 in an informal report. 2 Requirements that pertain to the radiation surveillance instrumentation for component performance were prepared in February 1973. Some surveillance and inspection techniques were considered primary whereas others were intended to supplement nonradiation techniques. The requirements read as follows:

a. Supplemental enrichment verification of declared UF 6 cylinders-simple, rapid, hand-held, inexpensive instrumentation that can distinguish (as a minimum) between feed, tails, product, and enrichments greater than product. The primary mode for verifying declared UF 6 cylinders was by nonradiation techniques using cylinder weights and mass spectrometer analysis of samples. In the secondary mode, active and passive neutron enrichment meters were used in situ to verify enrichment to a precision of about $10 \%$. Passive gamma-ray techniques for enrichment verification were also included.

b. Primary monitoring of sealed packages with an acceptable detection level for unauthorized uranium.

c. Supplemental monitoring of sealed packages--a quantitative measurement of material detected in item $b$. The monitoring and assay of packages were investigated in depth with a number of active and passive techniques. The detection sensitivity of the various methods was determined for packages specifjed in the reference model. However, the detection sensitivity depended on uncertain assumptions about package size and material content. Hence, a prototype package monitor was not constructed as part of the program.

d. Primary monitoring of personnel and vehicles--detection of unauthorized transport of uranium through portals with data storage capability, tamper-resistant and/or tamper-indicating equipment. A prototype, unattended personnel doorway monitor with the desired features was fabricated and tested at false alarm and detection levels consistent with national safeguards requirements. The additional design constraints imposed by long periods of reliable, unattended operation did cause a minor degradation in uranium sensitivity. In the case of vehicle portals to the restricted area, greater source-to-detector distances and the ability to use hidden shielding made it economically impractical to obtain detection levels comparable to the 
personnel doorway monitor. Hence, an unattended shipping dock monitor was designed and tested as a monitor for undeclared feed in significant quantities. An inspector could supplement this monitor with a sensitive, hand-held survey instrument for random checks of packages and vehicles.

Some development details of the radiation surveillance instrumentation were presented to representatives of the Tripartite Group at meetings held in April and September 1973. The group requested Los Alamos to loan specific enrichment meters (gamma and neutron) to the Almelo gas centrifuge facility for evaluation. Los Alamos personnel delivered the equipment in October 1974 and demonstrated its use.

\section{RADIATION CHARACTERISTICS OF URANIUM HEXAFLUORIDE}

The uranium enrichment techniques for both the gaseous diffusion and gas centrifuge methods require the uranium to be in the form of uranium hexafluoride $\left(\mathrm{UF}_{6}\right)$ in the process stream. This section summarizes the characteristic gamma-ray and neutron radiations from $U F_{6}$ for four common uranium isotopes that are present and significant for surveillance purposes. Alpha and beta radiation from the uranium isotopes and their daughter products generally do not penetrate the $U_{6} 6$ container walls and are not useful signatures.

\section{A. Gamma Radiation from Uranium Isotopes}

${ }^{234} \mathrm{U}$ lsotope. Weak gamma rays at 53.3 and $120.9 \mathrm{keV}$ are the only gamma signatures from ${ }^{234} \mathrm{U}$. The $53.3-\mathrm{keV}$ gamma ray generally will not escape from $\mathrm{UF}_{6}$ cylinders. The $120.9-\mathrm{keV}$ signature is strongly attenuated in the cylinder walls and cannot be resolved by $\mathrm{Nal}(\mathrm{Tl})$ detectors from the U-KB $x$-ray complex in the $110-$ to $115-k e V$ region.

$235 \mathrm{U}$ Isotope. The well known $185.7-\mathrm{keV}$ gamma ray from $235 \mathrm{U}$ occurs in about $55 \%$ of the alpha decays of this isotope. Weaker gamma rays at $143.8,163.4$, and $205.3 \mathrm{keV}$ are also characteristic of ${ }^{235} \mathrm{U}$. Other very weak gamma rays up to about $400 \mathrm{keV}$ maximum energy are found in the decay of $235 \mathrm{U}$. The $185.7-\mathrm{keV}$ gamma ray is commonly used to determine $235^{\mathrm{U}}$ enrichment, but is strongly self-absorbed in solid UF 6 . The mean free path of the 185.7-keV gamma ray in solid $U_{6}\left(5.1 \mathrm{~g} / \mathrm{cm}^{3}\right.$ density) is $1.9 \mathrm{~mm}$, which makes the material appear essentially as a surface source; measurement results indicate surface enrichment.

236 U Isotope. This isotope does not occur naturally and would not be present in an enrichment plant that used only natural uranium feed. It is present in the US enrichment system because material with a reactor history is recycled through the enrichment process. The weak, low energy $49.4-\mathrm{keV}$ gamma ray is not observed except from samples highly enriched in $236_{U}$. Uranium-236 does not provide a useful gamma-ray signature in materia! commonly found in the enrichment cycle.

$238 \mathrm{U}$ Isotope. Uranium-238 does not signature. Gamma rays from the $234 \mathrm{mPa}$ and identify ${ }^{238} \mathrm{U}$. The growth rate of these have a direct gamma-ray ${ }^{234} \mathrm{~Pa}$ daughters are used to daughters is the 24.1-day 
half-life of the intervening ${ }^{234} \mathrm{Th}$ in the following decay chain:

$238 \mathrm{U} \frac{4.47 \times 10^{9} \mathrm{y}}{\alpha} \rightarrow{ }^{234} \mathrm{Th} \quad \frac{24.1 \mathrm{~d}}{\mathrm{~B}^{-}} \rightarrow{ }^{234} \mathrm{~Pa} \quad \frac{1.17 \mathrm{~m}}{\mathrm{~B}^{-}} \rightarrow 234 \mathrm{U} \frac{2.44 \times 10^{5} \mathrm{y}}{\alpha} \ldots$

The characteristic gamma-ray energies used to identify $238 \mathrm{U}$ are 766.4 and $1001.0 \mathrm{keV}$ from $234 \mathrm{mPa}$. In equilibrium, these occur in 0.22 and $0.59 \%$ of the ${ }^{238} \mathrm{U}$ alpha decays, respectively. The spectrum also contains weaker gamma rays up to a maximum energy of about $2 \mathrm{MeV}$.

The time variation of these signatures in the first few months after chemical separation makes this a somewhat unreliable technique for ${ }^{2} 38_{U}$ assay in a uranium enrichment facility. The fluorides of the thorium and protactinium daughters are not volatile, thus vaporization during the enrichment process effects a chemical separation. To correlate $234 \mathrm{mPa}$ gamma-ray intensity with ${ }^{238} \mathrm{U}$ enrichment accurately after passage through an enrichment cascade, the age of the sample must be known if it is less than about 3 or 4 months.

B. Other Features in $U_{6}$ Cylinder Gamma-Ray Spectra

If the enrichment cascade has used feed recycled from a reactor, then additional gamma-ray lines can appear in the spectra of UF $_{6}$ product cylinders. Gamma rays from thorium daughters appear from small amounts of $232 \mathrm{Th}$ produced during reactor irradiation and enriched along with the lighter uranium isotopes in the separation cascade. The thorium daughters have principal gamma rays with characteristic energies of $511,583,727,860$, and $2614 \mathrm{keV}$. Other fission products and transuranics that are not completely removed during fiel reprocessing can contribute to the gamma-ray spectra from UF 6 cylinders. These contaminants will deposit and build up in UF $_{6}$ cylindians if they are not thoroughly cleaned between fillings. For highly enriched uranium (HEU), the $1274-\mathrm{keV}$ line from ${ }^{22} \mathrm{Na}$ of ten appears in the $\mathrm{UF}_{6}$ spectrum. This line arises from the ${ }^{19} \mathrm{~F}(\alpha, n){ }^{22} \mathrm{Na}$ reaction, and the intensity is time dependent, growing in with the $2.6-y$ r half-life of $22 \mathrm{Na}$.

C. Examples of Gamma-Ray Spectra of $\mathrm{UF}_{6}$ Cylinders

Examples of the gamma-ray spectra from $\mathrm{UF}_{6}$ cylinders in the US enrichment complex appear in Figs. 1 and 2. These spectra were accumulated using a high-resolution $\mathrm{Ge}(\mathrm{I} . \mathrm{i})$ detector. The headings in the figures give the identification number, cylinder type, ${ }^{*}$ and $235 \mathrm{U}$ enrichment on the first line; and fill mass, count time, and cylinder-detector distance on the second line.

Figure la is the $\mathrm{Ge}(\mathrm{Li})$ spectrum of an empty Type $30 \mathrm{~A}$ cylinder containing a nominal $4.5-\mathrm{kg}$ heel. ${ }^{* *}$ There is fairly intense radiation from deposited $234 \mathrm{Th}$ and $234 \mathrm{~Pa}$ daughters. Lines from the $137 \mathrm{Cs}$ fission product and ${ }^{23} \mathrm{~Pa}$ are also prominent. This is a typical dirty cylinder spectrum.

\footnotetext{
${ }^{*}$ Container nomenclature and cylinder types are described in Ref. 3. Appendix A gives a summary of sizes and weights.

${ }^{*} A$ heel is what remains in a UF $_{6}$ container that has been emptied but not cleaned.
} 


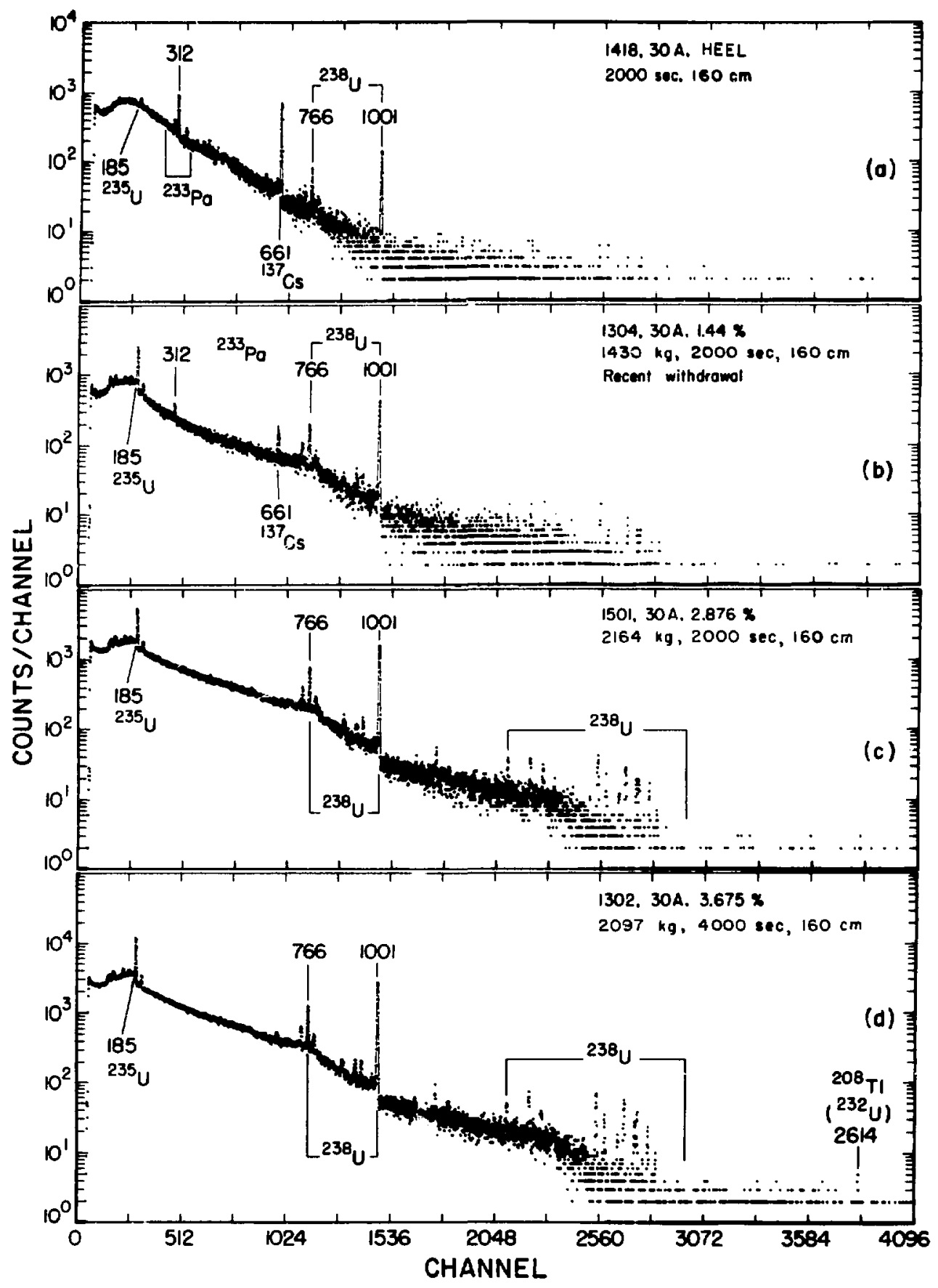

Fig. 1.

$\mathrm{Ge}(\mathrm{Li})$ gamma-ray spectra of empty and low-enriched Type 30A UF 6 cylinders. 


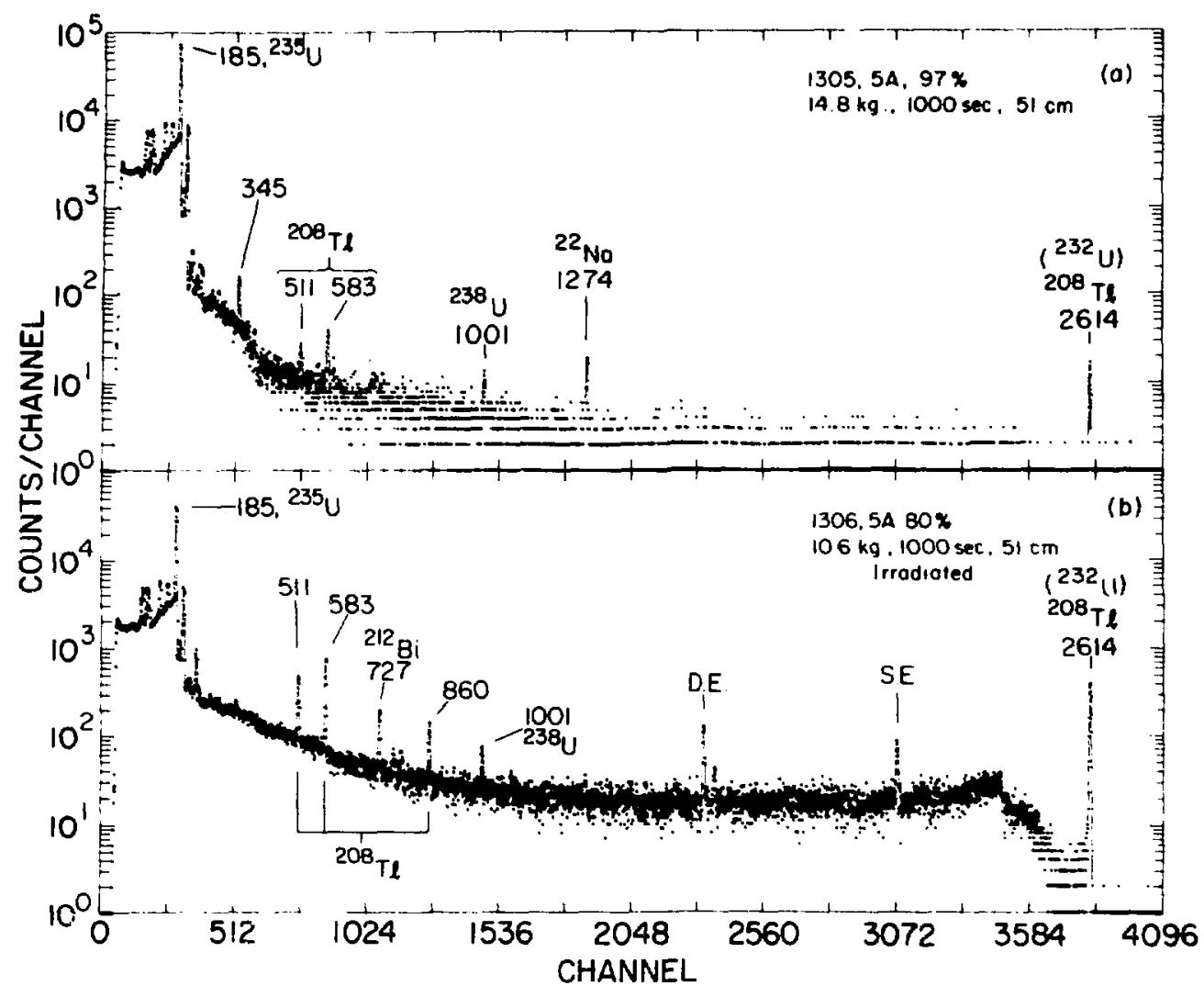

Fig. 2.

$\mathrm{Ge}(\mathrm{Li})$ gamma-ray spectra of two high-enriched Type $5 \mathrm{~A} \mathrm{UF}_{6}$ cylinders.

A comparison of parts $b$ and $c$ in Fig. I shows that both have atout the
same $238 \mathrm{U}$ content, but the smaller mass and more recent withdrawal of the filling in part $b$ give a less intense spectrum than that in part $c$. For these spectra, the detector viewed the entire cylinder, so that the count rate varies somewhat with the fill height and thus the fill mass. Some evidence of a dirty cylinder is also seen in part $b$.

In Fig. $2 \mathrm{a},{ }^{22} \mathrm{Na}$ appears. Gamma rays from the thorium daughter ${ }^{208} \mathrm{Tl}$ appear in both parts $\mathrm{a}$ and $\mathrm{b}$, and are especially prominent in the spectrum of part b. The material in this cylinder had a previous irradiation history. The $208 \mathrm{TI}$ gamma rays appeared in some low enrichment product cylinders (Fig. Id) and not in others (Fig. Ia, b, and c). This may be just an age difference. We did not expect to see $208_{\mathrm{Tl}}$ in natural feed or tails; spectra of those samples (not shown) confirmed its absence. 


\section{Neutron Output from $\mathrm{UF}_{6}$ Cylinders}

The neutron output from $U_{6}$ arises primarily from spontaneous fission by $23 S_{U}$ and from the ${ }^{9} \mathrm{~F}(\alpha, n)^{22} \mathrm{Na}$ reaction. The spontarieous fission neutron source strength is $15 \mathrm{n} / \mathrm{sec} \cdot \mathrm{kg}$ of $238 \mathrm{U}$. These neutrons, whose spectrum is shown in Fig. 3a, have an energy' spectrum typical of fission neutrons. Part b shows the $(\alpha, n)$ spectrum of an $241 \mathrm{Am}-\mathrm{F}$ source, ${ }^{4}$ which is similar to that expected from a uranium-fluorine source. Note the different energy scales. The actual neutron leakage spectrum from a large UF 6 cylinder will differ from that of Fig. 3 because of scattering in the UF 6 . Calculations have been made showing the effects of scattering on the leakage spectra from Type $30 \mathrm{~A}$ UF $_{6}$ cylinders. 5

The principal alpha emitter in enriched uranium is $234 \mathrm{U}$; the $234 \mathrm{U}$-produced neutrons dominate the $(\alpha, n)$ intensity from even low enriched ${ }^{235} \mathrm{U}$ product. The total neutron source term from a mass of UF 6 can be written 6 as

$Q=M_{U}\left(576 f_{34}+0.122 f_{35}+3.95 f_{36}+0.0279 f_{38}\right)$,

where $Q$ is the neutron source strength in $n / s e c, M_{u}$ is the uranium mass in $g$, and $f$ is the fractional isotopic composition of the subscripted isotope.
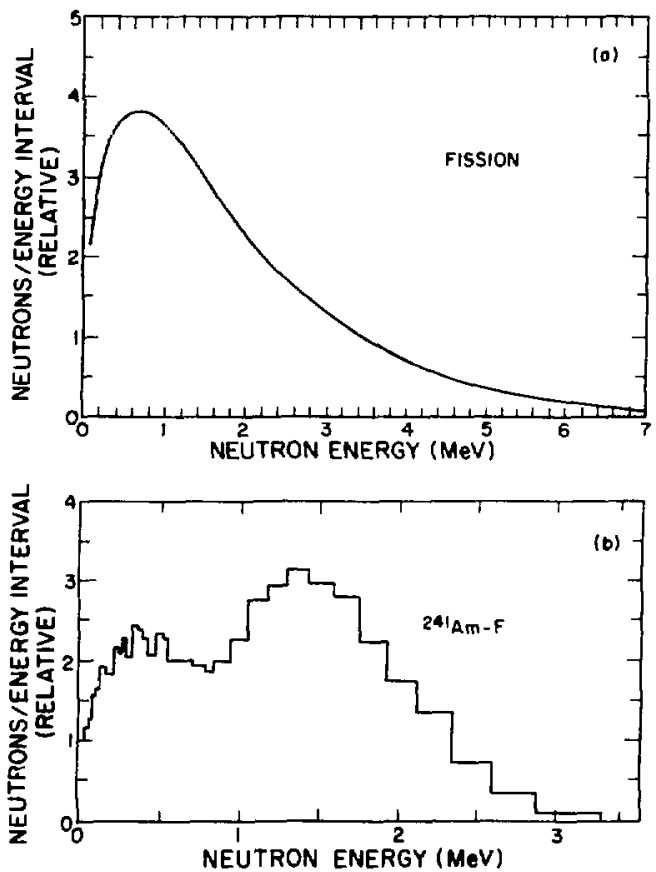

Fig. 3.

Neutron energy spectra: (a) fission source and

(b) $241 \mathrm{Am}-\mathrm{F}$ isotopic source. 
Equation ( 1$)$ inciudes both $(\alpha, n)$ and spontaneous fission contributions, but neglects any multiplication or absorption in the cylinder. The $238 \mathrm{U}$ term includes approximately equal contributions from spontaneous fission $(0.015 \mathrm{n} / \mathrm{sec} \cdot \mathrm{g})$ and $(\alpha, n)$ reactions $(0.013 \mathrm{n} / \mathrm{sec} \cdot \mathrm{g})$.

Neglecting $236 \mathrm{U}$, the relative contribution of the uranium isotopes to the total neutron yield is shown in Table I for three typical enrichments, as calculated from Eq. (1). The neutron source from depleted $U_{6} F_{6}$ arises chiefly from $238 \mathrm{U}$, whereas that for $3 \%$ enriched $U_{6}$ comes mainly from $234 \mathrm{U}$. The ${ }^{234} \mathrm{U}$ and $238 \mathrm{U}$ contributions are essentially equal for natural uranium.

\section{INSTRUMENTATION FOR PERIMETER SUR VEILLANCE}

\section{A. Personnel Doorway Monitor}

The personnel doorway monitor consists of an enclosure containing a detector array, signal conditioning electronics, power supplies, alarm logic circuits; occupancy monitor, alarm recording camera, and a tamper-indicating envelope. Design decisions, such as detector type and electronic circuit details, were based on work previously done to develop a personnel monitor for the national safeguards system. However, compromises were made to the monitor to accommodate tamper-proofing procedures. Frequent reference will be made to an earlier publication, Ref. 7 , for background information.

\section{TABLE I}

\section{RELATIVE CONTRIBUTIONS OF URANIUM ISOTOFES TO TOTAL NEUTRON YIELD}

\begin{tabular}{|c|c|c|c|c|}
\hline Description & Isotope & $\begin{array}{c}\text { Isotopic } \\
\text { Composition (\%) }\end{array}$ & $\begin{array}{c}\text { Neutron Yield } \\
\text { (n/sec g Uranium) }\end{array}$ & $\begin{array}{l}\text { Relative } \\
\text { Yield (\%) }\end{array}$ \\
\hline \multirow[t]{3}{*}{ Depleted } & ${ }^{234} \mathrm{U}$ & 0.0014 & $8.06 \times 10^{-3}$ & 22.2 \\
\hline & ${ }^{235} \mathrm{U}$ & 0.30 & $3.66 \times 10^{-4}$ & 1.0 \\
\hline & ${ }^{238} \mathrm{U}$ & 99.70 & $2.78 \times 10^{-2}$ & 76.7 \\
\hline \multirow[t]{3}{*}{ Natural } & ${ }^{234} \mathrm{U}$ & 0.0049 & $2.82 \times 10^{-2}$ & 49.7 \\
\hline & ${ }^{235} \mathrm{U}$ & 0.71 & $8.66 \times 10^{-4}$ & 1.5 \\
\hline & ${ }^{238} \mathrm{U}$ & 99.29 & $2.77 \times 10^{-2}$ & 48.8 \\
\hline \multirow[t]{3}{*}{ Product } & $234 \mathrm{U}$ & 0.025 & $1.44 \times 10^{-1}$ & 82.4 \\
\hline & & 3.00 & $3.66 \times 10^{-3}$ & 2.1 \\
\hline & ${ }^{238} \mathrm{U}$ & 96.98 & $2.71 \times 10^{-2}$ & 15.5 \\
\hline
\end{tabular}


Design guidelines for the doorway monitor specified that it would be unattended. Therefore, the monitor would have to record alarm events in such a way that it could identify an occupant; could detect and indicate tampering or other attempts at disabling the monitor function; and could operate independently of line power, if necessary, for short periods of time. The sensitivity guideline specified that the doorway should detect about $10 \mathrm{~g}$ of $235 \mathrm{U}$ in metallic compact geometry.

Sandia Laboratories used tamper-indicating techniques to protect the personnel doorway monitor. Several of the techniques provide unambiguous evidence of attempts to gain access to the instrumentation. The techniques differ in detail, but each requires disfiguring a visible surface to penetrate an enclosure for the purpose of tampering with the contents. Once the surface is disfigured, it is very difficult, if not impossible, to restore it. These tamper-indicating techniques are described in brief by Chambers and Ney. 8

The initial plan for the doorway monitor was that it consist of a single pole containing all detectors, electronics, power supplies, and camera in a simple, tamper-indicating package partly surrounded by gamma-ray shielding. The pole geometry did not allow room for large scintillators, herice small $\mathrm{NaI}(\mathrm{T} \ell)$ detectors were selected in a suitable size. ${ }^{7}$ The particular $\mathrm{NaI}(\mathrm{T} \ell)$ detector chosen (Fig. 4) has a scintillator in the shape of a 3.2- by 6.4- by $20.3-\mathrm{cm}$ rectangular prism that fits into the pole.

\section{Sensitivity measurements} made on a morkup of a singlepole doorway monitor using the $\mathrm{NaI}(\mathrm{Tl})$ detectors revealed two problems. Both resulted from the single-pole geometry: the background count increased when the monitor was occupied; moreover, the monitor was insensitive to material located on the far side of an occupant's body. In experiments where a person walked through the doorway monitor carrying $235 \mathrm{U}$ on the side away from the pole, the body shielded the source so that it was difficult to detect. We estimated that a 150 - to $300-g 235 \mathrm{U}$ source could be detected for $50 \%$ of passages. As a result, a second detector pole was incorporated, thus forming the conventional geometry for personnel monitors that detect special nuclear material (SNM).

The two-pole arrangement without a tamper-indicating enclosure appears in Fig. 5, where

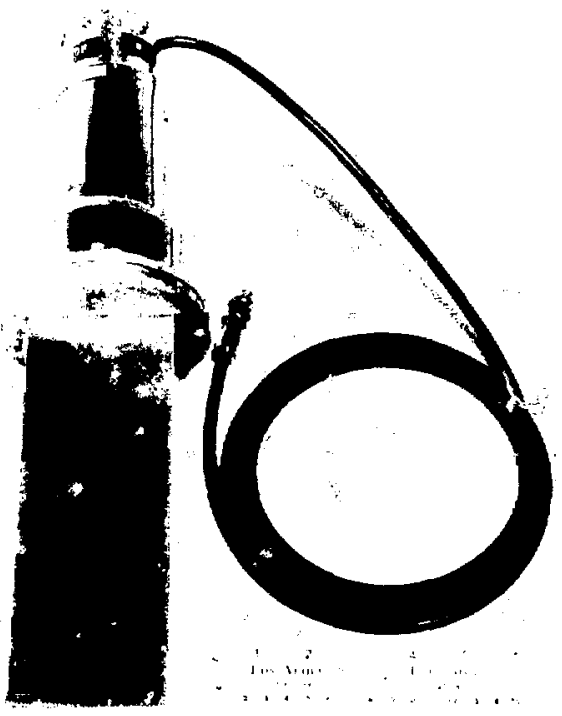

Fig. 4.

NaI(Te) detector for the personnel doorway monitor. 
some of the components are labeled. The four scintillators are staggered because the required detector positions in the electronics pole are not optimum. Some scintillator positions compensate for poor sensitivity in the waist and head regions. The camera and its associated optical train are located high on the pole to view the entire enclosure by means of the convex mirror at the top right of the figure. Just below the camera is the microwave occupancy monitor (unlabeled) used to control the framing rate of the camera, as described in a subsequent paragraph. The light fixtures provide proper illumiration for the camera. The poles are placed $76 \mathrm{~cm}$ apart; the distance from floor to ceiling is $190 \mathrm{~cm}$.

Figure 6 shows the component circuitry of the doorway monitor. The detector signal passes through a single channel analyzer (SCA), and we use both of its outputs: the lower-level discriminator (LLD) and the SCA window. The window is set for an eneigy range of approximately 60 to $250 \mathrm{keV}$, which is appropriate for detecting unshielded ${ }^{235} \mathrm{U}$ product. The lower-level discriminator passes all pulses greater than $60 \mathrm{keV}$, which is appropriate for detecting the higher energy spectrum of $238 \mathrm{U}$, the indicator of feed material. A sliding interval counter ${ }^{7}$ scales the output from each analyzer. A major interval of $1 \mathrm{sec}$ with four subintervals is used. A background count for each channel is separately accumulated over a time interval of 20 major intervals. The mean background plus four standard deviations $(4 \sigma)$ is used as an alarm level.

At each step of the sliding interval the count is compared to the alarm level. If the alarm level is exceeded while the doorway is occupied, the camera is enabled. If the doorway is unoccupied (a false alarm), the camera is not enabled until eight such alarms are accumulated. False alarms are handled in this manner in order to conserve film and yet provide a sufficient record of monitor operation.

An example of a film record appears in Fig. 7. The semitransparent mirror shown in Fig. 6 makes it possible to record on film the images of both the doorway occupant and a display of calendar and count rate infcrmation in each of the channels. The channel that alarmed is identified by decimal points that appear under the ratemeter display. The numerical information does not appear in the Fig. 7 example. 


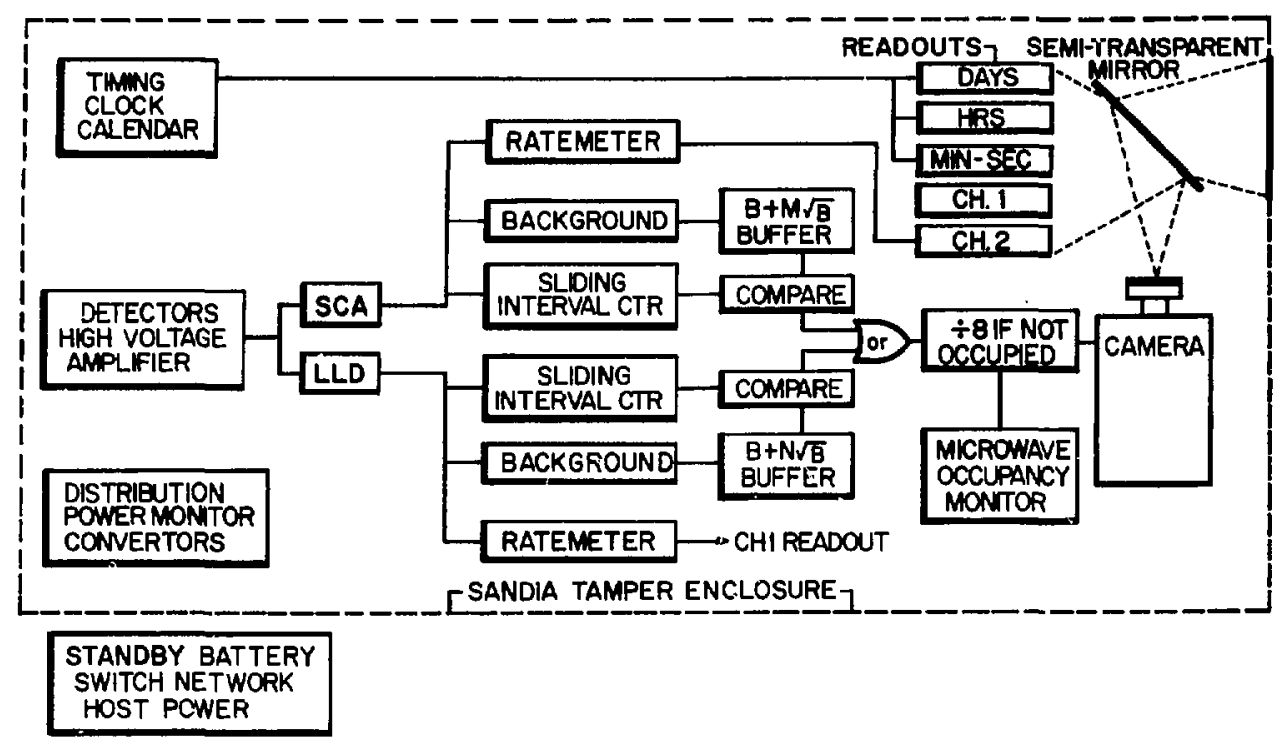

Fig. 6.

Personnel doorway monitor: logic diagram.

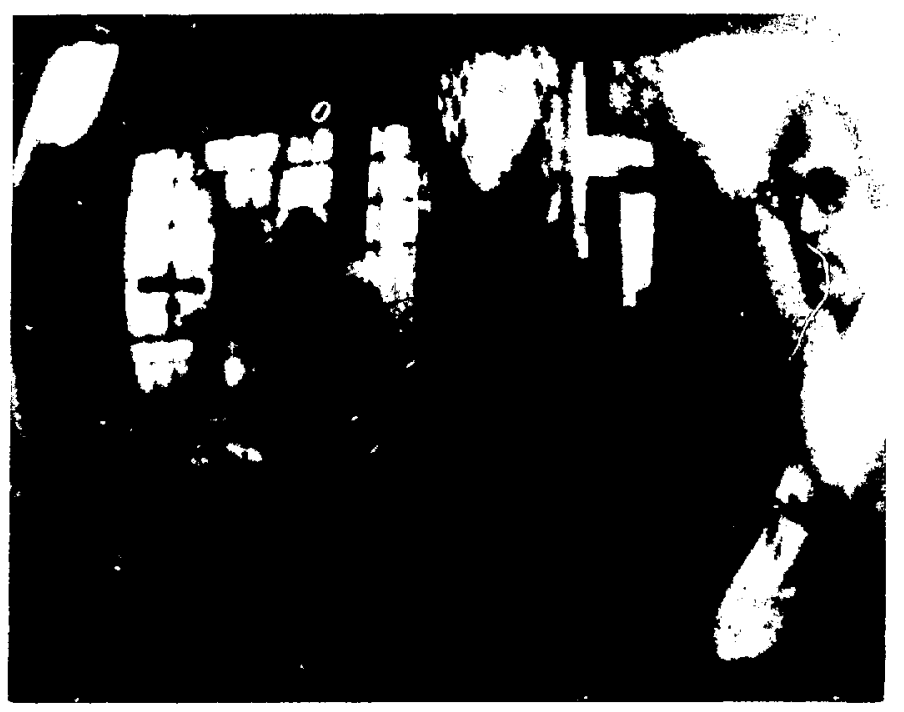

Fig. 7.

Personnel doorway monitor: example of film record. 
The complete monitor appears in Fig. 8. The glass tamper-indicating enclosures around the detector poles are screened to protect against accidental damage. A tamper-indicating connection in the pole bases requires a raised floor and ramp. The rest of the enclosure supports the mirror and lighting. The preferred direction of passage through the doorway monitor is in the counterclockwise direction, viewed from above, to obtain the best film images for identification. This should be the direction of passage for persons leaving the protected area. Clockwise motion produces photos of persons too near the mirror, which distorts their images, making it difficult to identify them.

Evaluation of the doorway monitor included a determination of the false alarm rate from statistical fluctuation in the counts and a separate determination of the monitor's sensitivity, obtained by having persons pass through carrying SNM sources. The false alarm rate was calculated using a computer model of the logic circuit and a Monte Carlo sampling technique. Figure 9 shows the results; the probability of an alarm is plotted against the background count duration. This alarm probability multiplied by the number of tests per hour gives the hourly alarm rate. At the background accumulation time used in the portal (20 major intervals), the expected alarm rate from statistical fluctuations would be $0.61 / \mathrm{h}$ per channel or $1.2 / \mathrm{h}$ for the two-channel logic package.

The most accurate experimental data for false alarms were obtained from film records. These observations were made overnight or over weekends; the intervals used were in the time period from the firsi: alarm after the end of

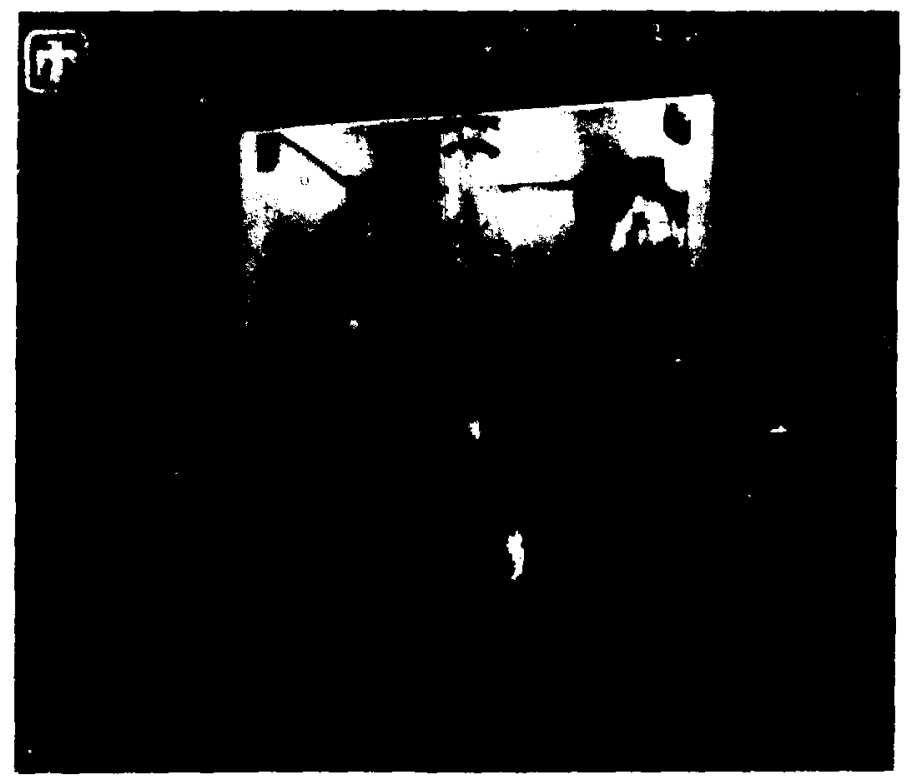

Fig. 8.

Personnel doorway monitor ready for use. 


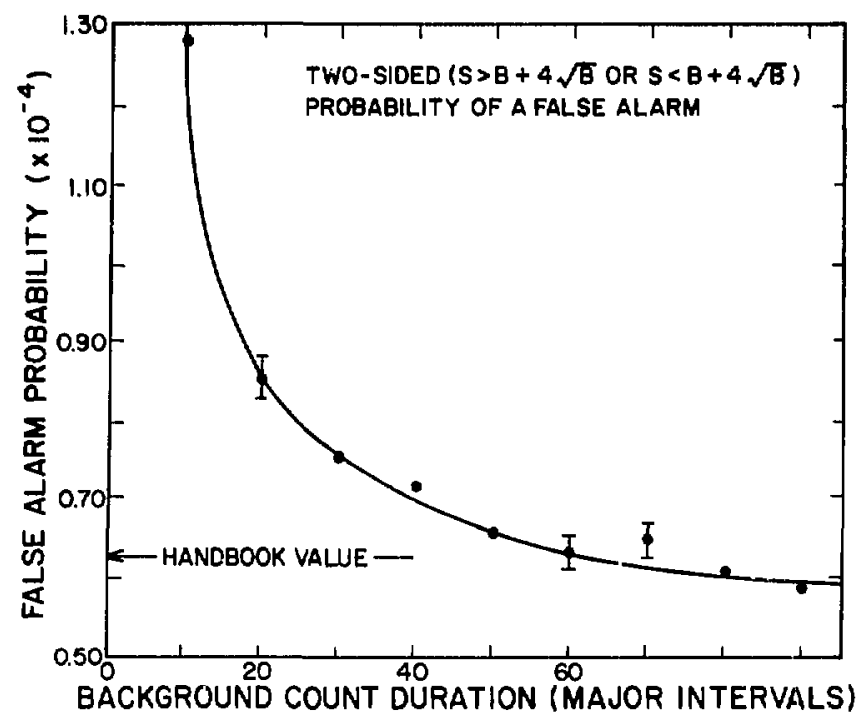

Fig. 9.

Calculated false-alarm probability versus background count interval.

normal working hours to the beginning of the next working day. The rate obtained from such records is 1.01 alarms/h over a time period of some $239 \mathrm{~h}$. This is somewhat less than the calculated rate, probab!; because the number of alarms used was eight times the number of film frames observed, and there would usually be a residual of less than eight accumulated alarms that could not be counted at the end of the overnight period. Using the sampling rate of the personnel monitor and the anticipated time required for passage, we expect that about I passage in 4000 will result in a false alarm.

The sensitivity of the doorway monitor for detecting $235 \mathrm{U}$ is determined in the same manner as for attended doorways. ${ }^{9}$ That is, the source is located on a person's body as he passes through the monitor. Before testing the monitor, we identified its regions of lowest sensitivity. The source size required for roughly $50 \%$ detection in the test regions was determined using eight different persons. These measurements, carried out in a $25-\mu R / h$ background, allow comparison between this and other doorway monitors that we evaluated. For this doorway monitor, the head and foot regions had the least sensitivity. For $50 \%$ detection, a person would have to carry a 30-g cylinder of uranium ( $93 \%$ enrichment, $27.4 \mathrm{~g} 235 \mathrm{U}$ content). This cylindrical uranium source was found to be equivalent to a 34-g HEU sphere $(32-\mathrm{g} 235 \mathrm{U}$ content, $93 \%$ enrichment, $99.75 \mathrm{wt} \%$ uranium) by laboratory measurement.

Because this was an unattended doorway monitor, additional tests were made. The source was held in outstretched hands at the farthest corners from a detector or similarly moved through distant corners when attached to a shoe. In this case, the result for sensitivity to HEU was $50 \mathrm{~g}$. 
After a period of laboratory evaluation and demonstration use, the personnel doorway monitor was sent to Winfrith Reactor Development Facility in the United Kingdom (UK) for operational testing. The results 10 of this test period indicated the need for some circuit debugging and some adaptation to background problems from plutonium at the reactor facility. An unfortunate outcome of this evaluation exercise was that irreparable damage occurred to the monitor in the return shipment to the US. The power supplies suffered severe salt water corrosion and the optical paths and scintillators suffered mechanical damage.

In retrospect, we would carry out the design of this monitor differently were it necessary to rebuild it. The original tamper-proofing constraint had resulted in a less-than-optimum detector array and custom-designed electronics. Recently, less complex means of tamper proofing have come into use, 11 allowing us to devote more room to detectors and electronics. Now we would use commercial equipment for signal processing and would select more optimum detectors. In particular, large plasiic scintillators properly placed would provide better detection of HEU and undeclared eed material, especially shielded feed material.

\section{B. Shipping Dock Monitor}

A shipping dock monitor was proposed to complement the personnel doorway monitor and to provide equal sensitivity for $235 \mathrm{U}$ detection. The shipping dock doorway was to be a 3-m-wide by 3-m-high opening with the monitor mounted above. The monitor was to consist of a single cylindrical enclosure that contained detectors, electronics and power supplies, logic circuits, occupancy monitors, and camera. For initial sensitivity measurements, this concept was mocked up with three and then four Nal(Tl) scintillators of the size used in the personnel doorway monitor. The sensitivity of the mockup, which did not have the gamma attenuation of a tamper-indicating enclosure, was estimated to be about $70 \mathrm{~g}$ of $235 \mathrm{U}$, in a $25-\mu \mathrm{R} / \mathrm{h}$ background, detected $50 \%$ of the time. The low sensitivity results primarily from the large opening that is monitored by a single set of detectors. Our assumption, in further developing the shipping dock monitor, was that it would only be used for detecting significant quantities of undeclared feed (UF 6 ).

In designing an instrument to monitor for undeclared feed, it is not necessary to make it sensitive to small amounts of SNM. Instead, introduction of undeclared feed in $1000-\mathrm{kg}$ quantities over a period of time must take place before there can be a diversion of significant quantities of HEU product. The principal gamma signature used for this type of monitor is the 1001.0 - and $766.4-\mathrm{keV}$ radiation from the $238_{\mathrm{U}}$ daughter, $234 \mathrm{~m} \mathrm{~Pa}$. Uranium-235 is present in low concentrations and contributes negligibly. The

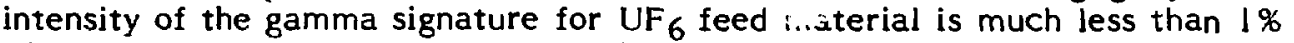
of the gamma signature for an equal mass of $\mathrm{HEU}$ considered in personnel monitoring. Nevertheless, large quantities of feed produce a detectable signal. In addition, there is neutron emission from the $U_{6}$ that may be detected. Although the neutron emission rate is low, backgrounds are also small, allowing us to consider both gamma and neutron detection for use in the shipping dock monitor. 
The gamma detector chosen for evaluation was a Nuclear Enterprises* NE 102 plastic scintillator, $51 \mathrm{~mm}$ in diameter by $914 \mathrm{~mm}$ long. Plastic was chosen primarily because the shipping dock monitor would experience rapid changes in temperature that might damage large $\mathrm{NaI}$ scintillators. For the evaluation, we used an SCA gamma-ray energy window from about 85 to 1000 $\mathrm{keV}$ and measured the count rate for metal spheres of depleted uranium and for small cylinders (Type IS, 400-g content) containing natural and depleted equilibrium UF6.

From the observed count rates for these sources (bare or shielded by $1.9 \mathrm{~cm}$ of lead), we estimated the required source count for $50 \%$ detection and determined, by a scaling method, the source that produces the required count rate. For metal spheres, the observed count rate $S$, during initial measurements, was consistent with a surface source, hence we used the scaling $S \propto$ mass $^{2 / 3}$. For the $U_{6}$ cylinders, it was necessary to calculate a geometrical scaling rule, using Type 15 and $5 \mathrm{~A}$ cylinder geometries. The nominal mass ratio of the two cylinder sizes as normally filled was known. The gamma-ray signal ratio was determined from the cylinder geometry and tabulated calculations of source geometry effects given by Rockwell. 12 The estimated sensitivity as scaled from reasurements at the center of the doorway on the floor is presented in Table Il.

\footnotetext{
* Nuclear Enterprises, Inc., 931 Terminal Way, San Carlos, CA 94070.
}

TABLE II

ESTIMATED SENSITIVITY OF A SHIPPING DOCK MONITOR CONTAINING A SINGLE PLASTIC ROD SCINTILLATOR

\begin{tabular}{lcc}
\cline { 2 - 2 } $\begin{array}{l}\text { Source Type } \\
\begin{array}{l}\text { Depleted uranium } \\
\text { metal sphere }\end{array}\end{array}$ & $\begin{array}{c}\text { Estimated 50\% Detection } \\
\text { Source }(\mathrm{kg})\end{array}$ & $\begin{array}{c}1.9-\mathrm{cm} \text { Lead Shielded } \\
\text { Source (kg) }\end{array}$ \\
$\begin{array}{l}\text { Natural UF6 } \\
\text { cylinder }\end{array}$ & 3 & 90 \\
$\begin{array}{l}235 \mathrm{U} \text { metal cylinder } \\
(93 \% \text { enriched) }\end{array}$ & 2.6 & 26 \\
\end{tabular}

aThe source materials were at equilibrium in $238 \mathrm{U}$ daughters. 
The neutron detector evaluated for the shipping dock monitor for $\mathrm{UF}_{6}$ feed was the very large Harshaw* model $\mathrm{B} 4-72 \mathrm{~S} / 50 \mathrm{BF}_{3}$ detector, which has a 14.6-cm diam, 1.83-m active length, and is filled to $50 \mathrm{~cm}$ of mercury pressure of $\mathrm{BF}_{3}\left(96 \%, 10_{\mathrm{B}}\right)$. The Harshaw model provides good detection capability for a relatively low cost. Other neutron detector arrays might provide better detectability, but funding and timing limited our testing of neutron detectors.

To evaluate the neutron detector for the shipping dock monitor, we made static measurements on a single detector with a 2-cm-thick by $24.5-\mathrm{cm}-0 . d$. by $226.5-\mathrm{cm}-1$ ong polyethylene moderator surrounding the electrostatic shield on the detector. The electrostatic shie!d is necessary because the cathode operates at $-4800 \mathrm{~V}$. A ${ }^{238} \mathrm{Pu}-\mathrm{LiF}$ neutron source, calibrated by the National Bureau of Standards (NBS), was used for the measurements. This source has an average neutron energy of about $1.1 \mathrm{MeV}$, which reasonably approximates the neutron spectrum from large masses of $\mathrm{UF}_{6}$. For determining detectable masses, we used a background count rate of 10 counts/sec, a 4- $\sigma$ detection level, and a 5-sec count time.

The source strength needed for detection at a distance of $3 \mathrm{~m}$ was determined from the calibrated source measurements. Then masses of various forms of detectable uranium and $\mathrm{UF}_{6}$ were calculated from the known neutron output of these forms. The results appear in Table III. The detector geometry made it highly sensitive to scattered neutrons, hence the count rate strongly depended on the environment. The range of values listed in Table III corresponds to low- and high-scatter environments.

\footnotetext{
* The Harshaw Chemical Company, Crystal \& Electronic Products Department, 6801 Cochran Rd., Solon, $\mathrm{OH} 44139$.
}

TABLE III

NEUTRON SHIPPING DOCK MONITOR: DETECTABLE MASS OF VARIOUS FORMS OF URANIUM-BEARING MATERIALa

\begin{tabular}{lc}
\multicolumn{1}{c}{ Form } & Mass (kg) \\
\hline Depleted uranium (metal) & $480-980$ \\
Depleted $\mathrm{UF}_{6}(0.2 \%)$ & $350-720$ \\
Natural $\mathrm{UF}_{6}(0.7 \%)$ & $190-380$ \\
$\mathrm{UF}_{6}$ product (3\%) & $60-120$ \\
$\mathrm{UF}_{6}$ product (30\%) & $12-25$
\end{tabular}

$a_{\text {Background }}=10$ counts $/ \mathrm{sec}, t=5 \mathrm{sec}$, detection level $=4 \sigma$, and detector-to-source distance $=3 \mathrm{~m}$. 
For a full size Type $30 \mathrm{~A} \mathrm{UF}_{6}$ cylinder, the approximate neutron source terms are given in Table IV. Attenuation factors ranging from 4 to 24 would be needed to reduce these source terms below the detectability levels of Table III. For example, $13 \mathrm{~cm}$ of cadmium-covered polyethylene will give an attenuation by a factor of 5 for this neutron spectrum; in order to tightly surround a Type $30 \mathrm{~A}$ cylinder would require over $700 \mathrm{~kg}$ of cadmiumpolyethylene shielding. These figures show that the neutron detector would provide a satisfactory monitor for undeclared feed, but its sensitivity would be significantly worse than that estimated for gamma detection in Table II. Therefore, we developed a gamma monitor for the shipping dock monitor.

Having decided to use the plastic scintillator gamma-ray detector, we accommodated its length in one of the glass enclosures used for the personnel doorway monitor by modifying the electronics package so that the boards are behind the plastic scintillator rod, as shown in Fig. 10. Redesign of the electronics was required to accommodate a plastic scintillator; the amplifier needed to accept fast pulses from the plastic scintillator and a general noise reduction was necessary to allow setting the LLD jow enough to make use of the small pulses that are so profuse in plastic scintillators.

The logic diagram for the shipping dock monitor appears in Fig. 11. Although the monitor only uses an SCA LLD signal, it maintains two channels. The first channel is for signal alarms where a 4- $\sigma$ alarm level is used. The second channel alarms if the monitor is shielded to prevent its normal function. The shielding alarm level is set at $12 \sigma$ below the normal background. This feature exists because the monitor's low false alarm rate provides few photographs. Moreover, the optical system does not look directly at the detector pole, which makes subversion invisible. In the personnel monitor a statistical alarm produces a photo of any visible tampering within an hour. Should the shipping dock monitor be incapacitated by outside shielding, the second channel alarm records the fact on film by a series of film frames with alarms in the second channel.

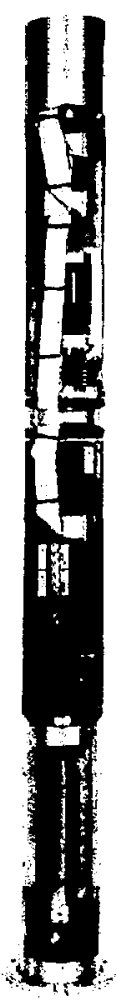

Fig. 10.

Shipping dock monitor: plastic scintillator and electronics package. 
TABLE IV

NEUTRON SOURCE TERMS FOR TYPE 30A UF 6 CYLINDERS

$\mathrm{UF}_{6}$ Enrichment

Depleted (0.2\%)

Feed (0.7\%)

Product (3\%)
Full Cylinder Neutron

Source $(n / \mathrm{sec})$

$4.1 \times 10^{4}$

$7.7 \times 10^{4}$

$2.4 \times 10^{5}$

The background update time in the logic for the shipping dock monitor remains at 20 major intervals, with the major interval still divided into 4 subintervals. The length of the major interval for the shipping dock monitor is $4 \mathrm{sec}$, in contrast to the $1-\mathrm{sec}$ interval used for the personnel monitor. This change is based on dynamic measurements analyzed using variable logic paraneters. The results in Fig. 12 indicate that most passes can be detected in the 4-sec major interval.

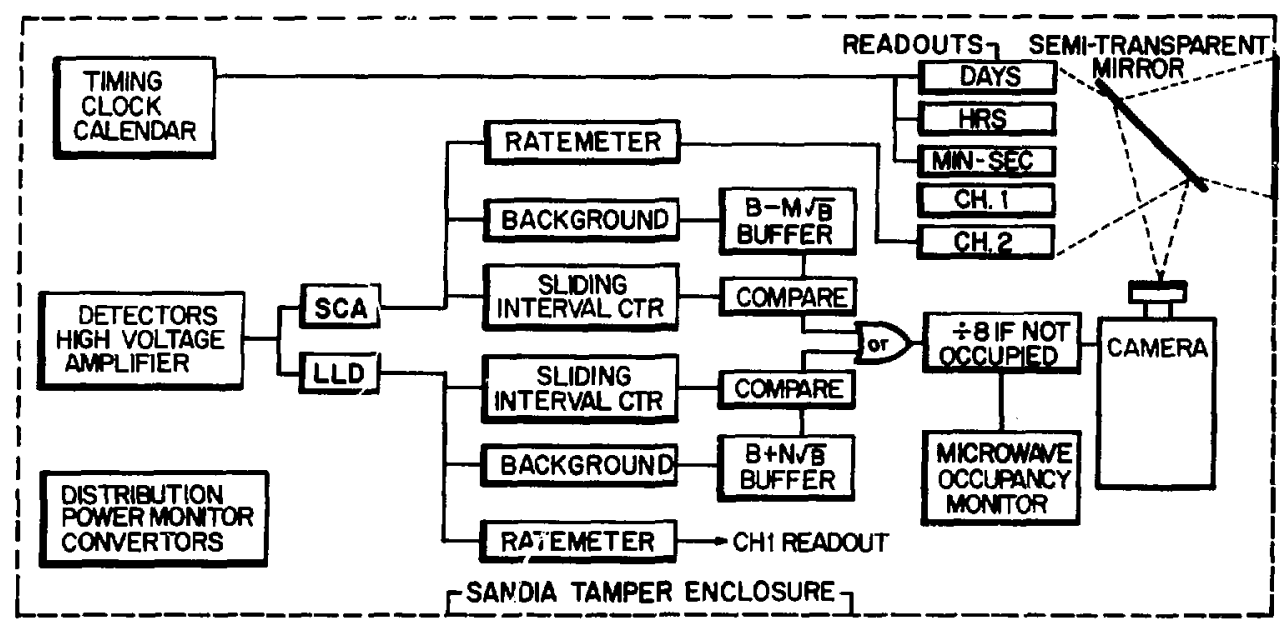

STANDBY BATTERY

SWITCH NETWORK

HOST POWER

Fig. 11 .

Shipping dock monitor: logic diagram. 


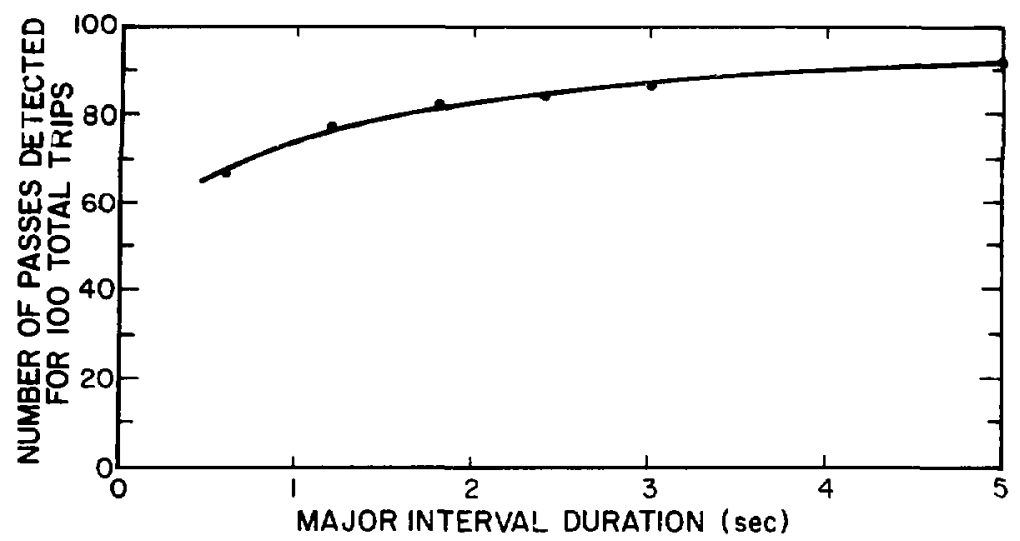

Fig. 12.

Results of measurements to determine optimum length of major time interval.

The hourly false alarm rate for the shipping dock monitor is reduced because the alarm rate for the 12- $\sigma$ channel is negligible and the 4- $\sigma$ channel samples at one-fourth the rate of the personnel monitor. The statistical alarm rate is thus one-eighth of the two-channel persornel monitor rate, or 0.15 alarms $/ \mathrm{h}$.

The shipping dock monitor appears in its final form in Fig. 13. The location of the detector is beside the door instead of above it, as initially envisioned, to make the installation more universal.

No field testing of the shipping dock monitor took place. Its low sensitivity to HEU made it unsuitable for domestic use. Other opportunities for field tests required specific energy response that was not readily available with the plastic scintillator.

In retrospect, the same reduced sensitivity in the personnel portal existed in the shipping dock monitor, both caused by the tamper-proofing constraint. Yet another problem was photomultiplier gain, which was less than required because, at the time, the well regulated high-voltage supply we needed was not available. Again, with more accommodating tamper-proofing techniques, better performance could be achieved.

C. Hand-Held Personnel, Package, and Vehicle Monitor

We developed a hand-held SNM monitor (Fig. 14) suitable for supplemental searching of personnel, vehicles, or packages to provide a complementary instrument for the personnel and shipping dock monitors. The instrument uses a small $\mathrm{NaI}(\mathrm{Tl})$ scintillation detector and battery-powered electronics. A logic circuit is used to derive an alarm test level that, when exceeded, results in the sounding of an audible alarm. A more detailed account of the monitor may be found in other reports. 13,14 


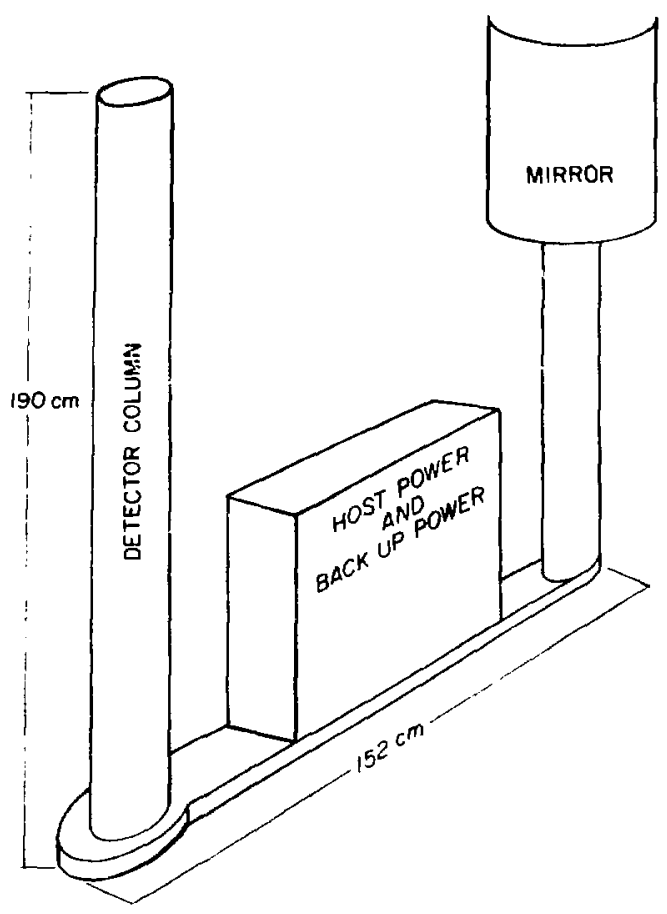

Fig. 13. Shipping dock monitor.

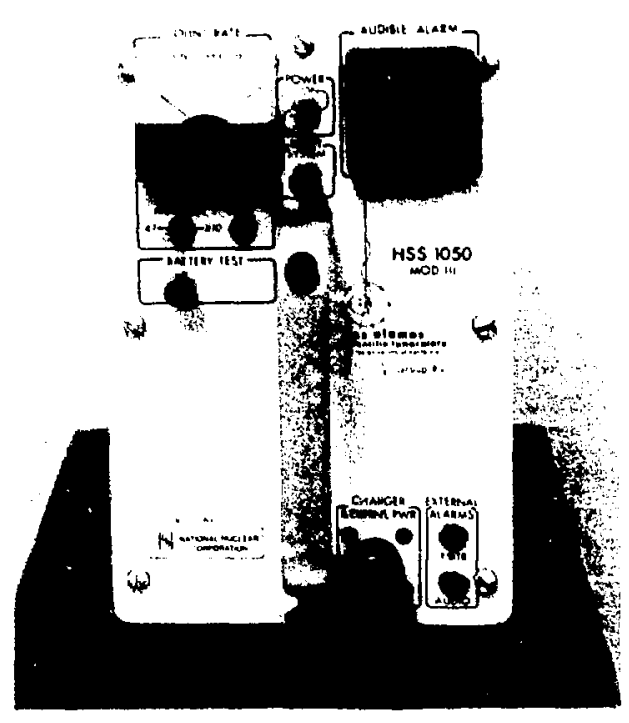

Fig. 14.

Hand-held SNM monitor for personnel, vehicles, and packages.

Tests of the monitor's sensitivity were performed using a $10 \mathrm{mg}$ spherical $235 \mathrm{U}$ source that was moved past the monitor at a speed of $0.5 \mathrm{~m} / \mathrm{sec}$. At a distance of closest approach equal to $0.15 \mathrm{~m}$, the detection probability was greater than $95 \%$. Thus, in a personnel search, an operator can do an adequate job by moving the instrument from head to toe four times at different places spaced evenly around the person's body, taking 4 to $6 \mathrm{sec}$ for each head to toe scan. In vehicle and package searches, all doors must be opened and access covers removed to permit the monitor to move through as much of the contained space as possible.

\section{Package Monitor}

In a model separation plant, boxes containing contaminated centrifuge parts for disposal or complete centrifuges needing repair continually cross the perimeter. For proprietary reasons, these boxes cannot be opened for visual inspection, which might reveal design information. Therefore, a means for determining whether the boxes contain HEU, clandestine and well shielded, is another part of an effective perimeter safeguards system. The basic problem is to distinguish HEU from ${ }^{238} \mathrm{U}$ and its daughters present in the package. The technique must also satisfy design requirements of modest cost, portability, 
limited radiation exposure to personnel, and ease of operation. It must be nonintrusive into protected centrifuge design information.

We considered many techniques for potential monitors, 'including $x-r a y$ radiography, neutron radiography, neutron transmission, active neutron interrogation, and passive gamma and neutron counting. We examined each technique to determine effectiveness "of bare and shielded HEU, with the following results.

\section{Passive Neutron Detection}

The large size of the centrifuge in the reference model ${ }^{2}$ and the low ineutron output from small quantities of $\mathrm{UF}_{6}$ or metallic uranium make the passive neutron approach unsatisfactory for this application. We measured no neutron signal above background for packages containing over $100 \mathrm{~g}$ of $\mathrm{UF}_{6}$ using moderated ${ }^{3}$ He detectors.

\section{Passive Gamma-Ray Detection}

Passive gamma-ray measurement of packages containing UF 6 or metallic uranium does provide an acceptable signal, but the technique can be easily foiled if the contents are shielded. Therefore, we went on to look at active techniques that are isss susceptible to shielding.

\section{Radiography}

Standard radiography can complement passive gamma-ray measurement by detecting shielding material or large amounts of UF 6 inside thick steel layers. To evaluate radiography for sealed classified packages, we radiographed wooden boxes containing mockup centrifuge parts. We used both Kodak* Industrex AA film and Kodak Industrex Instant 600 paper film with $90-\mathrm{keV} \times$ rays. In addition to mockup rotors and casings, the boxes contained uranium foils and uranium cubes of various sizes to determine a threshold of visibility for the uranium in the presence of the intervening shielding matter. Foils as thin as $0.13 \mathrm{~mm}$ and cubes as small as $1 \mathrm{~g}$ were usually easily detected. One disadvantage to $x$-ray and gamma-ray radiography is that the high-quality radiograph also reveals a great deal of information about the items in the package. A second disadvantage is that the radiograph does not uniquely identify high-density materials.

A possible way of identifying uranium uses $x$ rays filtered by a uranium $K$-edge absorption filter for a second raoiograph. The transmission of uranium varies markedly across the $115.6-\mathrm{keV} \mathrm{K-edge} \mathrm{where} \mathrm{the} \mathrm{transmission} \mathrm{of} \mathrm{iron}$ changes slowly. A change should be seen where uranium is present; however, our tests did not show sufficient contrast to be useful.

\footnotetext{
*Eastman Kodak Co., 1187 Ridge Road West, Rochester, NY 14650.
} 
Two gamma-ray sources of widely differing energies can also be used to obtain a contrast for uranium detection. Mass attenuation coefficients for high-energy gamma rays (over $\mathrm{I} \mathrm{MeV}$ ) are almost independerit of atomic number $z$, whereas at lower energies these coefficients increase rapidly with $z$. Two radiographs are required. We used ${ }^{192} \mathrm{Ir}(316-\mathrm{keV})$ and $60 \mathrm{Co}(1.17-$ and I.33-MeV) gamma rays, which produced good results; however, the technique is still intrusive.

\section{Neutron Radiography}

Another poteritial solution to the problem of finding metallic $235 \mathrm{U}$ in the presence of propri tary centrifuge parts contaminated with $238 \mathrm{U}$ is to use a neutron source to radiograph the box and its contents. This method takes advantage of the high thermal neutron absorption of $235 \mathrm{U}$ relative to $238 \mathrm{U}$ and other materials in the package.

A neutron source of $7 \mu \mathrm{g}$ of ${ }^{252} \mathrm{Cf}$ suitable for package interrogation is easy to handle and is inexpensive. The source $\left(1.7 \times 10^{7} \mathrm{n} / \mathrm{sec}\right)$ must be moderated to increase the thermal neutron component of the neutron spectrum. With this source, our exposure times were long--about $250 \mathrm{~h}$ using a $6_{\mathrm{LiF}} \mathrm{ZnS}$ screen and Polaroid 3000 spced film. The film-screen combination was the most sensitive arailable. The resolution we obtained was the size of the $5-\mathrm{cm}$-diam source moderator and allowed as much as a kilogram of metallic uranium to go undetected. Other problems that we experienced included scattering by iron and hydrogen present in the package that reduced the contrast of the image.

\section{Neutron Transmission}

Further investigation of neutron scattering and the effectiveness of neutron interrogation in identifying $235 \mathrm{U}$ led us to try a thermal neutron detector for neutron transmission measurements of attenuation and scattering. We used a $7-\mu \mathrm{g}{ }^{252} \mathrm{Cf}$ source, moderated by $5 \mathrm{~cm}$ of polyethylene, and a ${ }^{3} \mathrm{He}$ collimated proportional counter for minimum room-scatter signal. We decided to use a source-detector separation of $0.9 \mathrm{~m}$ and placed 1 -mm-thick $235 \mathrm{U}$ foils in the neutron beam. Using this configuration, we could easily detect foil masses from 2 to $15 \mathrm{~g}$. A plywood box containing a mockup aluminum centrifuge rotor placed between the neutron source and the detector substantially reduced the sensitivity by scattering neutrons into the detector. A mockup steel centrifuge in the box completely masked the presence of all foils by scattering.

\section{Active Neutron Interrogation Using Delayed Neutrons}

We used delayed fission neutrons for detection of HEU or ${ }^{238} \mathrm{U}$ in an experiment with a Cockcroft-Walton $300-\mathrm{keV}$ accelerator source of $14-\mathrm{MeV}$ neutrons $\left[{ }^{3} \mathrm{H}(\mathrm{D}, \mathrm{n})^{4} \mathrm{He}\right.$ reaction] and a small slab neutron detector (sixteen $2.5-\mathrm{cm}$-diam by $91-\mathrm{cm}-\mathrm{long}{ }^{3} \mathrm{He}$ tubes in a $7.6 \mathrm{-cm}-$ thick slab of polyethylene). For the experiment, we operated the accelerator at a repetition rate of ten $5-10 \mathrm{msec}$ duration pulses/sec. The detector preamplifier was gated off for $50 \mathrm{msec}$ before each pulse, and the actual counting time was 
$50 \mathrm{msec}$ after the pulse. The detector was positioned $1.22 \mathrm{~m}$ from the accelerator target. Figure 15 shows the net delayed neutrons detected as we varied the position of a package containing a 987-g sample of $93 \% 235 \mathrm{U}$ between the source and detector. The figure shows a least sensitive position roughly halfway between the neutron source and the detector.

Using that least sensitive position, we measured a number of samples. A steady-state background, primarily from cosmic rays, was measured with the accelerator off. Background caused by accelerator dark current was measured with gating in operation.

The results shown in Table $V$ for ${ }^{23} 5_{U}$ and ${ }^{238} U$ are sample averages for three runs with background subtracted. The uncertainty $\sigma$ can be expressed as

$\sigma=\left[-\frac{1}{3}\left(\begin{array}{c}\text { average } \\ \text { total count }\end{array}\right)+\frac{1}{3}\left(\begin{array}{l}\text { average back }- \\ \text { ground count }\end{array}\right)\right]^{1 / 2}$,

where the average background count is 1436 per $100 \mathrm{sec}$ determined from three runs. Column 5 relates these measurements to a potential field system

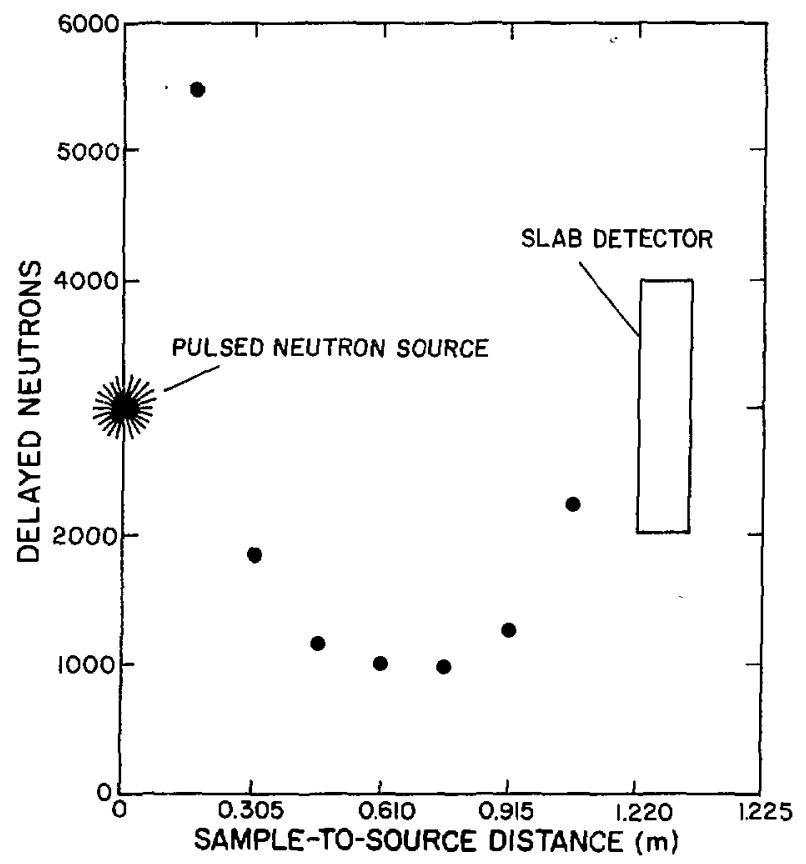

Fig. 15.

Delayed fission neutrons detected for different package positions. 
using a Kaman Sciences Corporation* mobile generator that produces 14-MeV pulsed neutrons from a sealed tube. The generator produces $1.0 \times 10^{8}$ neutrons per pulse at a rate of 10 pulses/sec. This source can be operated with a shorter $20-\mathrm{msec}$ detector preamplifier off-gate before each pulse, giving a 1.6 increase in counting time. Figure 16 shows the expected Kaman delayed neutron count. The results for ${ }^{235} \mathrm{U}$ deviate from a straight line mainly because of neutron multiplication. We estimated the uncertainty $\sigma=30$ in Fig. 16 and show $3 \sigma$ above background where the sensitivity is $300 \mathrm{~g}$ of $235 \mathrm{U}$ or $238 \mathrm{U}$. The sensitivity may be improved to $100-150 \mathrm{~g}$ of $235 \mathrm{U}$ or $238 \mathrm{U}$ with pulsed high voltage in the Kaman tube to reduce dark currents. When we included a centrifuge casing mockup inside the plywood box package containing ${ }^{235} \mathrm{U}$ or ${ }^{238} \mathrm{U}$, the results were essentially the same as in Table $\mathrm{V}$.

*Kaman Sciences Corporation, P. O. Box 7463-T, Colorado Springs, CO 80933.

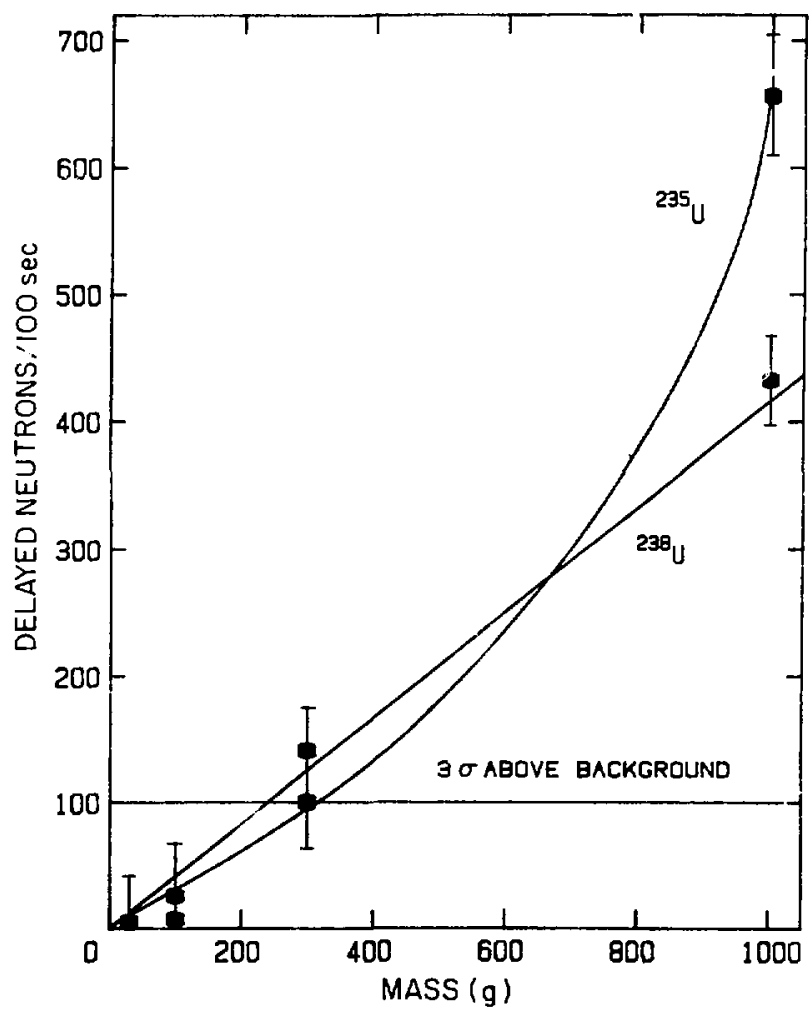

Fig. 16.

Estimated delayed neutron count with a field system. 
TABLE $V$

DELAYED NEUTRONS FROM SAMPLES OF 238 U AND $235 \mathrm{U}$

\begin{tabular}{|c|c|c|c|c|}
\hline Sample (g) & $\begin{array}{l}\text { Average } \\
\text { Total } \\
\text { Counts a } \\
\end{array}$ & $\begin{array}{l}\text { Net Delayed } \\
\text { Neutrons }\end{array}$ & $\begin{array}{c}\text { Uncertainty } \\
\sigma\end{array}$ & $\begin{array}{l}\text { Delayed Neutrons } \\
\text { Expected for } \\
\text { Kaman Sciences } \\
\text { Neutron Generatorc } \\
\end{array}$ \\
\hline $1002.6\left({ }^{238} U\right)$ & 2107 & 671 & 34 & 429 \\
\hline $300.55\left(^{238} U\right)$ & 1655 & 219 & 32 & 140 \\
\hline $100.18\left({ }^{238} U\right)$ & 1454 & 18 & 31 & 11 \\
\hline $987.1593 *\left({ }^{235} \mathrm{U}\right)$ & 2457 & 1021 & 36 & 653 \\
\hline $295.0593 \%\left({ }^{235} \mathrm{U}\right)$ & 1594 & 158 & 31 & 101 \\
\hline $98.5993 \%\left({ }^{235} U\right)$ & 1480 & 44 & 3] & 28 \\
\hline $29.4993 \%\left({ }^{235} \mathrm{U}\right)$ & 1447 & 11 & 31 & 7 \\
\hline
\end{tabular}

a Neutron source provides $2.5 \times 10^{8} \mathrm{n} / \mathrm{pulse}$ and $10 \mathrm{pulses} / \mathrm{sec}$. Detector preamp gated off for $50 \mathrm{msec}$ before each pulse. All data are averages of three $100-\mathrm{sec}$ runs with $50 \mathrm{msec} / \mathrm{pulse}$ counting time.

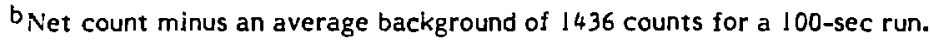

$\mathrm{c}_{1} 0^{8} \mathrm{n} / \mathrm{pulse}, 10 \mathrm{pulses} / \mathrm{sec}$, and $80 \mathrm{msec} / \mathrm{pulse}$ counting time.

The presence of moderating material (polyethylene) placed between the source and $235 \mathrm{U}$ or $238 \mathrm{U}$ sample decreased the signal. We were able to detect a signal through up to $30 \mathrm{~cm}$ of moderator thickness. Polyethylene between the sample and the detector caused a larger signal loss; the addition of $0.8 \mathrm{~mm}$ of cadmium foil to $5 \mathrm{~cm}$ of moderator completely shielded the sample.

\section{Active Neutron Interrogation Lsing Prompt Fission Neutrons}

Prompt fission neutrons can be detected with ${ }^{4} \mathrm{He}$ proportional counters or other fast neutron detectors, and the measurement can be used to detect the presence of fissionable material. The idea is to interrogate a package with low-energy neutrons that can induce fission in $235 \mathrm{U}$ but do not fission $238_{\mathrm{U}}$ (whose threshold is about $1 \mathrm{MeV}$ ) and do not exceed the detection threshold of the ${ }^{4} \mathrm{He}$ counters (whose threshold is about $0.5 \mathrm{MeV}$ ).

Our initial experiments used a moderated ${ }^{252} \mathrm{Cf}$ fission source (of average energy $\overline{\mathrm{E}} \sim 2 \mathrm{MeV})$, a moderated $(\alpha, n)$ neutron source $\left({ }^{238} \mathrm{Pu}-\mathrm{Li}, \overline{\mathrm{E}} \sim\right.$ $0.5 \mathrm{MeV})$, and three nearly monoenergetic $(\gamma, n)$ photoneutron sources ( ${ }^{24} \mathrm{Na}-\mathrm{D}, \overline{\mathrm{E}} \sim 265 \mathrm{keV}$; ${ }^{226} \mathrm{Ra}-\mathrm{Be}, \overline{\mathrm{E}} \sim 90 \mathrm{keV}$; and $\left.{ }^{2} 24 \mathrm{Sb}-\mathrm{Be}, \overline{\mathrm{E}} \sim 24 \mathrm{keV}\right)$. The photoneutron sources gave the best signal-to-background ratio and, of these, the ${ }^{124} \mathrm{Sb}-\mathrm{Be}$ was best and was used in an experiment that inspected mockup packages.

The package used in our inspection trial was built of $1-\mathrm{cm}$-thick plywood and contained a $1-\mathrm{cm}$-thick steel casing in which we placed samples of metallic HEU. Our high-purity, natural ${ }^{24} \mathrm{Sb}$ gamma-ray source had an 
intensity of $2.5 \mathrm{Ci}$ of $1.69-\mathrm{MeV}$ activity and was contained in 10-cm-thick beryllium and a $15-\mathrm{cm}$-thick lead shield. The detector used four $5.1-\mathrm{cm}$ by 66-cm-long 18-atm ${ }^{4} \mathrm{He}$ counters schematically shown in the experiment sketch in Fig. 17. The counters were shielded with boral ${ }^{*}$ to reduce background in the counters from $(n, \alpha)$ reactions in small amounts of $10_{B}$ present as an impurity in the counter wall.

Our results are summarized in Table VI where the ratio $S / B^{1 / 2}$ is used to indicate the detected signal $S$ in units of standard deviation of the background $\left(\sigma=\mathrm{B}^{1 / 2}\right)$. A reasonable detection threshold is $3 \sigma$ and a signal of that size corresponds to about $50 \mathrm{~g}$ of $235 \mathrm{U}$ shown in the table. Figure 18 displays the results plotted against the surface area of each sample rather than its mass. The resulting straight line demonstrates that interrogation is effective on the sample surface only. The results in Table VI also demonstrate a projected area dependence for a disk source. The surface area dependence is an important drawback for the technique because a small amount of uranium plating can give a positive indication equal to a much larger mass of clandestine $\mathrm{HEU}$, thus increasing the detection threshold for the technique.

${ }^{*}$ A Boron-aluminum material used to absorb neutrons.

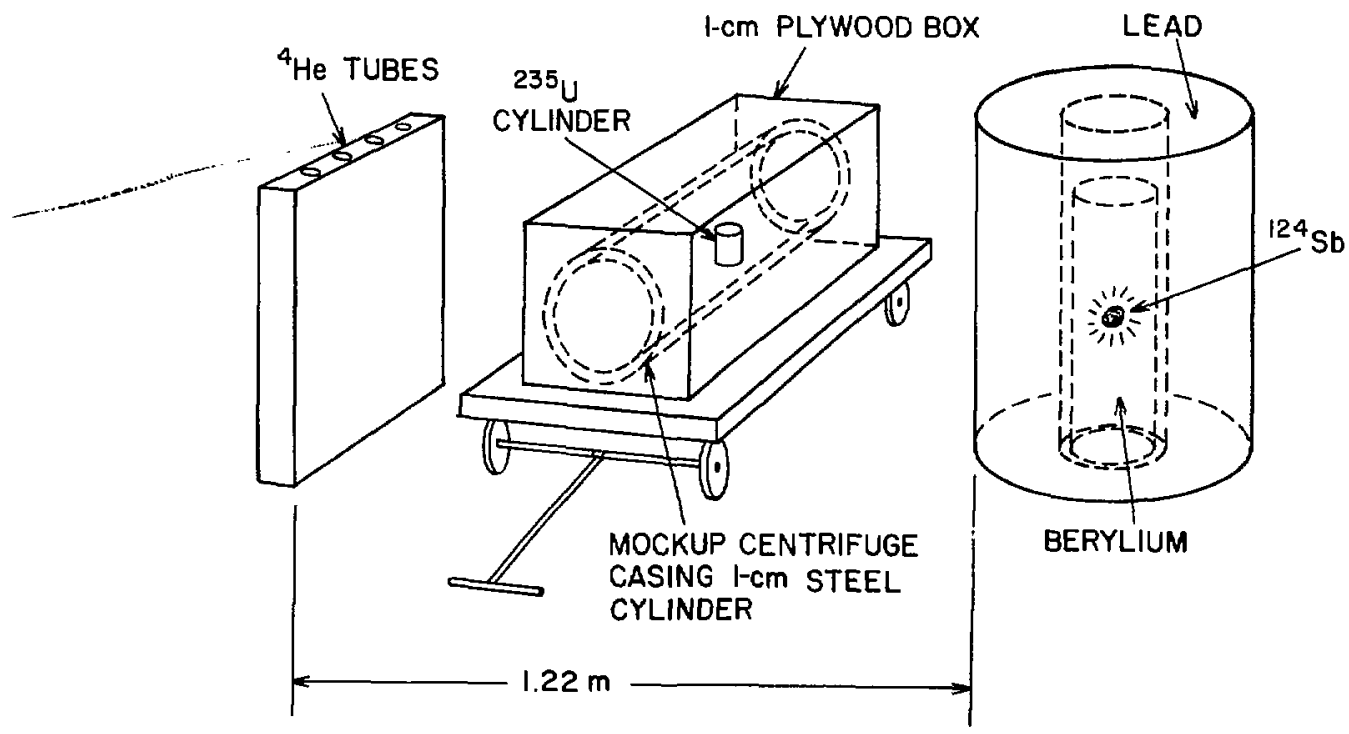

Fig. 17.

Experiment for measuring prompt fission neutron response using $\$ 24 \mathrm{Sb}-\mathrm{Be}$ source interrogation. 
PROMPT NEUTRON RESULTS FOR 235U SAMPLE INTERROGATIONa

\begin{tabular}{|c|c|c|}
\hline 235U Sample & $S /(B)^{1 / 2}$ & Net Counts \\
\hline 1-kg disk, 3-mm thick, horizontal & 66.6 & 773 \\
\hline 1-kg disk, 3-mm thick, upright & 138. & 1601 \\
\hline $1-k_{g}$ cylinder ${ }^{b}$ & 21.6 & 251 \\
\hline 300-g cylinder & 9.9 & 115 \\
\hline 100-g cylinder & 4.5 & 52 \\
\hline 30-g cylinder & 1.9 & 22 \\
\hline 10-g cylinder & 0.95 & 11 \\
\hline
\end{tabular}

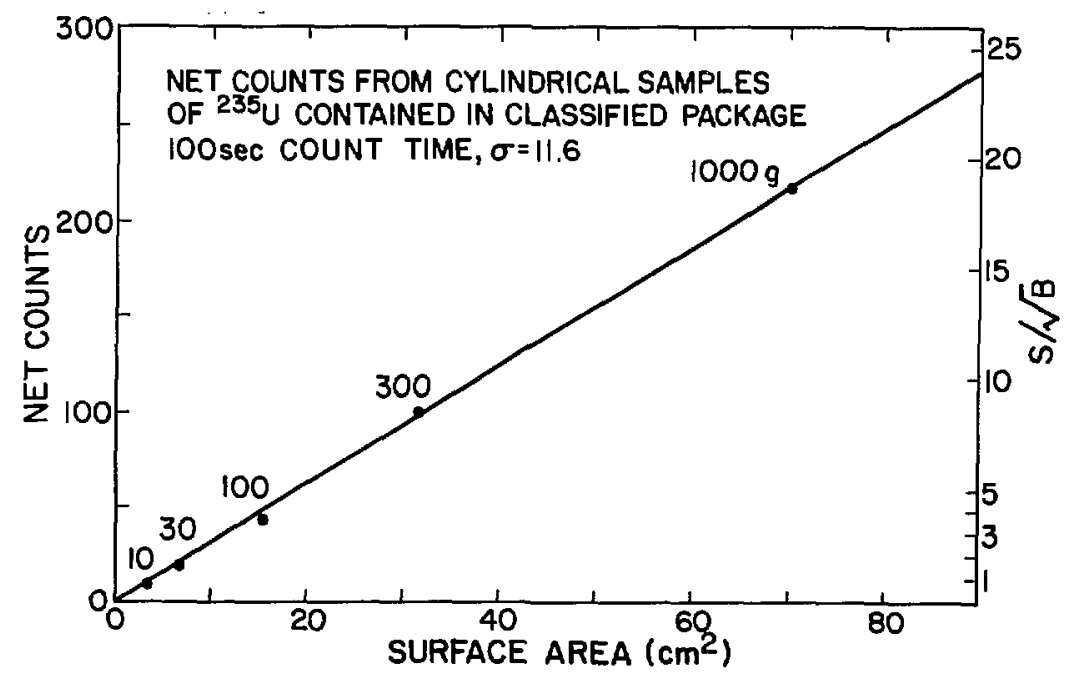

Fig. 18.

Package monitor response linearity with sample surface area. 
Limitations to this technique are caused by the effectiveness of neutron shielding material on the interrogation by low-energy neutrons. The effects of cadmium foil, polyethylene, or combinations of the two are summarized in Table VII. For our 100-sec counting period, $50 \mathrm{~mm}$ of polyethylene are sufficient to reduce the signal from $100 \mathrm{~g}$ of $235 \mathrm{U}$ below the 3- $\sigma$ threshold for detection. Morever, the same sample wrapped in cadmium, with one-half that polyethylene thickness, is effectively shielded from the interrogating neutrons.

To summarize, all of the package monitoring techniques suffer from intrusiveness or susceptibility to neutron or gamma-ray shielding. We made no attempt to pursue package monitoring further. Recently, neutron interrogation followed by fast neutron detection was used by Smith and Rice ${ }^{15}$ for package monitoring in SNM diversion safeguards. Kunz proposes the same technique for classified waste monitoring. ${ }^{16}$ The technique still depends on thermal neutron interrogation and is susceptible to shielding. The shielding detection scheme incorporated by Smith and Rice is not effective for all shielding configurations.

TABLE VII

SHIELDING RESULTS USING THE PROMPT NEUTRON TECHNIQUEa

${ }^{235}$ U Sample

1-kg disk, 3-mm thick

I-kg cylinder

300 g cylinder

$100 \mathrm{~g}$ cylinder

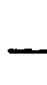

$0.5-\mathrm{mm}$ cadmium

no shielding

$0.5-\mathrm{mm}$ cadmium

50-mm polyethylene

cadmium foil $+50-\mathrm{mm}$ polyethylene

$75-\mathrm{mm}$ polyethylene

no shielding

75-mm polyethylene

no shielding

25.4-mm polyethylene

cadmium foil $+25.4-\mathrm{mm}$ polyethylene

50-mm polyethylene

cadmium foil $+50-\mathrm{mm}$ polyethylene

$63.5-\mathrm{mm}$ polyethylene

$76.2-\mathrm{mm}$ polyethylene
$\mathrm{S} / \mathrm{B}^{1 / 2}$

60.5

26.0

16.8

9.5

1.1

3.3

22.2

1.2

5.5

8.2

1.0

2.25

0.

1.8

0.4

${ }^{\mathrm{a}}$ Counting period of $100-\mathrm{sec}, \mathrm{B}=135$ counts. 


\section{PORTABLE INSTRUMENTS FOR VERIFYING URANIUM ENRICHMENT}

Verification techniques are intended to permit an independent measurement of uranium enrichment in $\mathrm{UF}_{6}$, which is contained primarily in large cylinders. Verification is not an exact assay; instead it is a technique that determines a result within about $10 \%$ of the true enrichment. We examined neutron and gamma-ray techniques for verification. The techniques used hand-carried instruments described in the following paragraphs for both laboratory and field measurements.

\section{A. Gamma-Ray Enrichment Meter}

The gamma-ray enrichment technique uses the intensity of the $185.7-\mathrm{keV}$ gamma ray from $235 \mathrm{U}$ as a measure of the $235 \mathrm{U}$ enrichment in a sample. The 186-keV gamma-ray activity from a thick, uniform, uranium-bearing object will be proportional to the enrichment of $235 \mathrm{U}^{1} 17$ For solid $\mathrm{UF}_{6}$, the mean-free path of the $186-\mathrm{keV}$ gamma ray is $1.9 \mathrm{~mm}$; thus, only those gamma rays that originate in the outermost few millimeters of the UF 6 will escape from the cylinder, and the technique views only the surface of the $\mathrm{UF}_{6}$ filling. Normally, the isotopic enrichment is uniform throughout the cylinder; however, if subversion can take place, the lack of penetrability is an important drawback.

$\mathrm{NaI}(\mathrm{Tl})$ gamma-ray detectors with portable battery-powered electronics are commercially available as enrichment meters. Ultrahigh-purity germanium detectors are coming into use and offer energy resolution that solves the dirty cylinder problems mentioned in Sec. II-B that can thwart a $\mathrm{Na}(\mathrm{Tl})$ detector. We have only used $\mathrm{Nal}(\mathrm{Tl})$ instruments in the tests reported here.

Measurements of ${ }^{235} \mathrm{U}$ enrichment with portable $\mathrm{NaI}(\mathrm{Tl})$ detectors require two energy windows. One window is set to bracket the $186-\mathrm{keV} 235 \mathrm{U}$ peak; counts in this window represent the $186-\mathrm{keV}$ signal plus the background underlying the peak. The second window is set just above the peak to determine the background alone. With the proper calibration constants a and $b$ for count rates in the two windows, the expression for $235 \mathrm{U}$ enrichment I can be written as

$I=a C_{A}-b C_{B}$

where $C_{A}$ and $C_{B}$ are observed counts in the peak window and background window, respectively, and $a$ and $b$ are calibration constants obtained from measurement of two known standards.

A commercially available instrument, Eberline Instrument Corporation's* Stabilized Assay Meter II (SAM-II), performs the two-channel analysis. The SAM-II unit consists of a $5.1-\mathrm{cm}$-diam by $1.27-\mathrm{cm} \mathrm{NaI}(\mathrm{Tl})$ detector and a battery-powered electronics package. The detector contains a 241 Am alpha

\footnotetext{
${ }^{*}$ Eberline Instrument Corporation, P. O. Box 2108, Santa Fe, NM 87501.
} 
particle source to provide a constant energy pulse for electronic stabilization. The electronics consist of a stabilized high-voltage supply and two separate amplifier-SCA channels. The unit has an up-down scaler that performs the subtraction of $\mathrm{Eq}$. (2). With an optional digital rate multiplier (DRM), the constants $a$ and $b$ can be preset and the result of Eq. (2) is the enrichment. The calibration constants $a$ and $b$ are determined from measurements of two samples of known enrichment. The count in each channel is recorded for each measurement, giving two equations in two unknowns:

$I_{1}=a C_{A}\left(I_{1}\right)-b C_{B}\left(I_{1}\right)$ and

$I_{2}=a C_{A}\left(I_{2}\right)-b C_{B}\left(I_{2}\right)$.

These equations are solved for $a$ and $b$ whose values are dialed into the DRM to calibrate the instrument.

Some problems occur in obtaining good calibration constants. The counts $C_{A}$ and $C_{B}$ should be measured at several places on the calibration cylinders to test uniformity. Dirty cylinders will usually yield widely differing results, depending on position. Large voids or cracks in the $\mathrm{UF}_{6}$ can result in low readings, and negative constants have been observed. Variable cylinder-wall thicknesses can cause errors in the calibration or the measurements. 17 The wall thicknesses vary not only between cylinder types, but also for the same cylinder.

The gamma-ray enrichment technique using the SAM-II instrument is in fairly general use in the US. The accuracy of the technique is about $10 \%$, because of cylinder wall variations, $U_{6}$ nonuniformity (such as cracks and voids), and unrecognized dirty cylinders.

Typical results that we obtained from measuring 15 Type $30 \mathrm{~A}$ product cylinders at the Portsmouth Gas Diffusion Plant are plotted in Fig. 19. Three cylinders, of the group of eighteen that were measured, have been omitted from these data because they gave inconsistent results for varying detector positions.

\section{B. An Alternate Gamma-Ray Method Using $235 \mathrm{U}$ and $238_{\mathrm{U}}$ Decay Radiation}

Some drawbacks to the single gamma-ray determination described in the preceding section can be avoided by using gamma rays from decay of both $235 \mathrm{U}$ and $238 \mathrm{U}$. The technique uses the $186-\mathrm{keV} 235 \mathrm{U}$ gamma ray with either the $766-$ or $1001-\mathrm{keV}$ gamma ray from the $234 \mathrm{~m} \mathrm{~Pa}$ daughter of $238 \mathrm{U}$. A relationship is derived in Appendix $B$ between enrichment and the ratio of, for example, a measured 186-keV count to a measured 1001-keV count $\left(\mathrm{C}_{186} / \mathrm{C}_{1001}\right)$. Figure 20 shows the relationship between measured count ratios and enrichment.

As a field test of this gamma-ray technique, we obtained $\mathrm{Ge}(\mathrm{Li})$ detector count ratios $\mathrm{C}_{186} / \mathrm{C}_{1001}$ for groups of $\mathrm{UF}_{6}$ cylinders at the Portsmouth Gas Diffusion Plant. Curves were calculated for each cylinder type to determine enrichment. We compared measured to actual enrichment to correct 


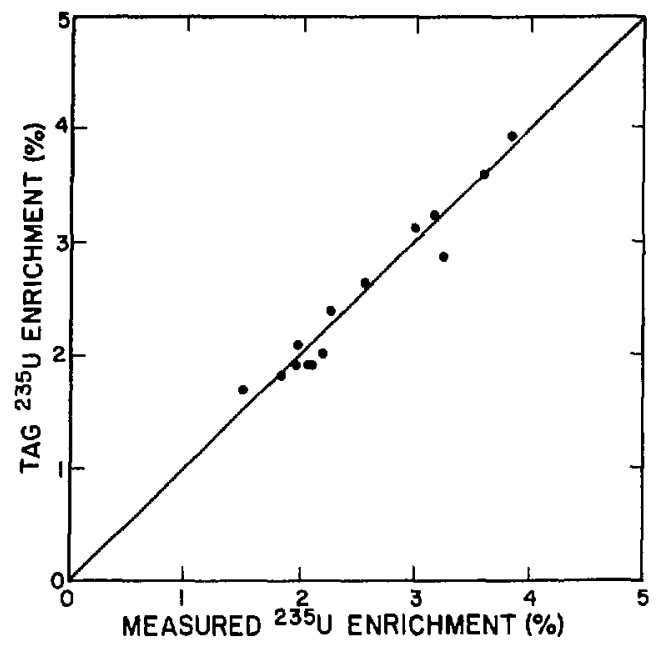

Fig. 19.

SAM-II gamma-ray enrichment measurements of Type $30 \mathrm{~A} \mathrm{UF}_{6}$ product cylinders.

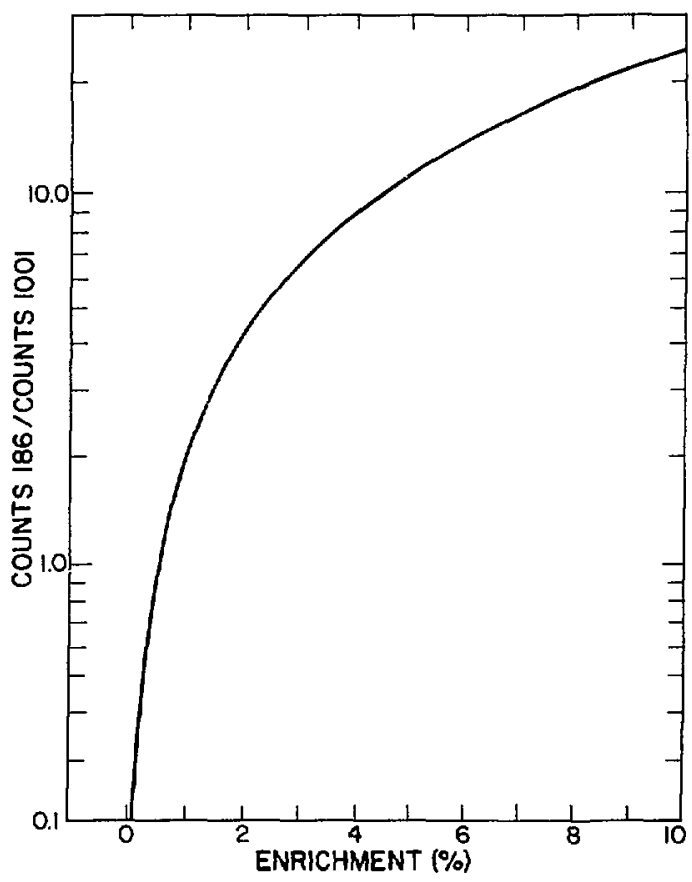

Fig. 20.

Ratio of 186-keV to 1001-keV gamma-ray counts as a function of enrichment for Type 5A cylinders using a small $\mathrm{NaI}$ detector. 
the efficiency parameter $\varepsilon_{186 / \varepsilon_{1001}}$ used in the calculation, which was not precisely known prior to the measurements. Table VIII compares the final measured enrichments to the actual values.

A second trial of the two gamma-ray technique was done at Los Alamos using small samples of $U_{6}$ that covered a wider range of enrichment. The agreement between measured and actual enrichment is shown in Fig. 21 for these Type IS cylinders. Part of the point scatter is caused by variation in thickness of the cylinder walls that introduces errors in $\mathrm{C}_{186} / \mathrm{C}_{1001}$ of up to $5 \%$.

In measuring $\mathrm{C}_{186} / \mathrm{C}_{1001}$, the counting time, distance from the detector, wall thickness, and diameter varied for each cylinder type. Only wall thickness affects the results for different cylinders. Counting time does not affect the count rates nor do geometrical effects. Only the ratio of intrinsic efficiencies affects the count rates. Cylinder diameter is not important for cylinders of Type 5A and larger. A consideration in applying this technique is that the 1001-keV gamma-ray activity builds up with the 24.1-day half-life of ${ }^{234} \mathrm{Th}$. Thus, in measuring $\mathrm{C}_{186} / \mathrm{C}_{1001}$ in $\mathrm{UF}_{6}$ cylinders, one must either be assured that about 3 months have elapsed since the cylinder fill date, or that the fill date is known approximately so that the measured ratio may be corrected. Another consideration is to make measurements at different places on the cylinder to detect variable results that may indicate dirty cylinders whose plating is thick enough to attenuate the $186-\mathrm{keV}$ radiation or whose activity is high enough to alter the shape of the gamma-ray spectrum.

\section{C. $\mathrm{UF}_{6}$ Enrichment Verification with a Thermal Neutron Detector}

The neutron source term for $\mathrm{UF}_{6}$, discussed in Sec. II and calculated in E.q. (1) 8 can be used for $U_{6}$ enrichment verification in several ways depending on the information at hand.

TABLE VIII

COMPARISON OF MEASURED AND ACTUAL VALUES OF ENRICHMENT FOR A GROUP OF UF $_{6}$-FILLED CYLINDERS

$\begin{array}{cccccc}\begin{array}{c}\text { Cylinder } \\ \text { Type }\end{array} & \begin{array}{c}\text { Measured } \\ C_{186} / \mathrm{G}_{1001}\end{array} & \begin{array}{c}\text { Measured } \\ \text { Enrichment (\%) }\end{array} & \begin{array}{c}\text { Actual } \\ \text { Enrichment (\%) }\end{array} & \text { Error (\%) } \\ 5 \mathrm{~A} & 4.19 & 3.94 & & 3.754 & 5 \\ 5 \mathrm{~A} & 4.81 & 4.47 & & 4.095 & 9 \\ 12 \mathrm{~A} & 5.23 & 4.45 & & 4.633 & 4 \\ 12 \mathrm{~A} & 11.40 & 9.28 & & 9.86 & 6 \\ 30 \mathrm{~A} & 2.10 & 2.33 & & 2.867 & 19 \\ 48 \mathrm{~A} & 0.534 & 0.85 & & 0.7108 & 20 \\ 48 \mathrm{~A} & 1.49 & 2.37 & & 2.478 & 4\end{array}$




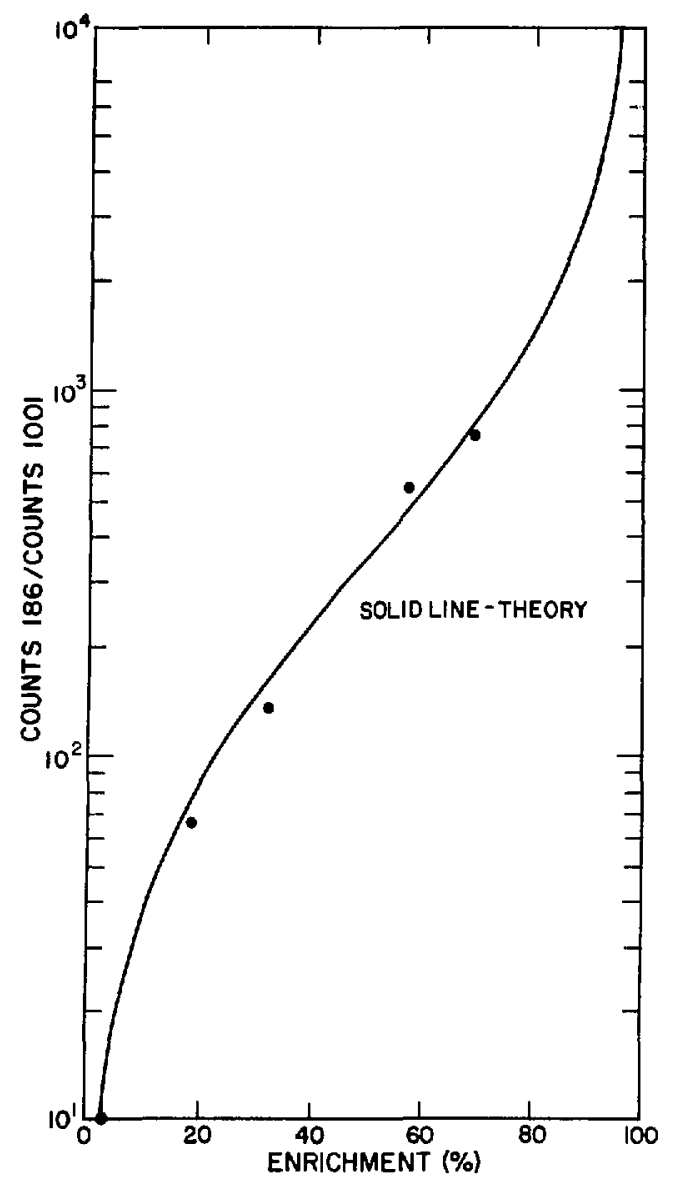

Fig. 21 .

Ratio of 186-keV to $1001-k e V$ gamma rays for Type $1 \mathrm{SUF}_{6}$ sample cylinders as a function of enrichment.

Case 1. When complete isotopic data are available and the $\mathrm{UF}_{6}$ mass is stated, comparison of the observed neutron count with the source strength calculated from Eq. (1) provides a method of verifying the consistency of the stated data for a set of cylinders. However, two offsetting deviations from stated tag values may produce the same result and the deviations would not be detected. Furthermore, the technique is not a direct measure of the $235 \mathrm{U}$ content.

Case 2. For common uranium isotopic compositions, the Eq. (1) terms $\mathrm{f}_{35}$ and $\mathrm{f}_{36}$ contribute little and can usually be neglected. Then the Eq. (1) expression for $Q$ can be approximated as

$Q \simeq M_{U}\left(576 f_{34}+0.0279 f_{38}\right)$. 
A comparison of the observed count rate with $Q$ in Eq. (4) can be made from the stated sample mass and $234 \mathrm{U}$ and $238 \mathrm{U}$ content. As with Case 1 , verification of the consistency of these three parameters by neutron counting does not yield direct information about $235 U$ content nor, in practice, is a knowledge of the ${ }^{234} \mathrm{U}$ content always available.

Case 3. Equations (1) and (4) show that passive neutron counting is not directly sensitive to $235 \mathrm{U}$ content. However, for well-behaved separation cascades, especially over the relatively small range of enrichment for light-water reactor ( $L$ W) fuel, the $235 U / 234 U$ ratio is essentially constant. Designating the ${ }^{235} \mathrm{U} /{ }^{234} \mathrm{U}$ isotopic ratio by $\mathrm{R}$, Eq. (4) becomes

$Q \simeq M_{U}\left(\frac{576 f_{35}}{R}+0.0279 f_{38}\right)$.

Replacing $f_{38}$ by $1-f_{35}$, the expression simplifies to

$Q \simeq M_{U}\left[\left(\frac{576}{R}-0.0279\right) f_{35}+0.0279\right]$

that has the form

$Q \simeq M_{u}\left(a+b f_{35}\right)$

Assuming that the $235 / 234$ ratio is either known or is constant, a neutron count may then be compared to $\mathrm{Q}_{3}$ calculated from Eq. (7), to provide an independent verification of the $2.35 \mathrm{U}$ mass and enrichment. Standard cylinders can be used to obtain the calibration constants $a$ and $b$.

This discussion assumes that the measured count is proportional to the neutron source strength $\mathrm{Q}$. This is not obviously true, given the large extended source of $\mathrm{UF}_{6}$ in a typical 2-1/2-ton product cylinder. However, factors affecting the assumption for moderated ${ }^{3} \mathrm{He}$ thermal neutron detectors, discussed in Ref. 18, seem to cancel each other, making the agreement between measured count and calculated $Q$ better than might be expected.

For our application of the thermal neutron technique, a hand-carried instrument using a moderated ${ }^{3} \mathrm{He}$ thermal neutron detector and commercial portable electronics, described by Walton and Atwell, 19 was redesigned to make it smaller, lighter, and less expensive at some sacrifice in efficiency. The modified shielded neutron assay probe (SNAP) detector (Fig. 22) weighed $7 \mathrm{~kg}$. The detector's back angle shielding factor is 2.4 for 238 PuLiF neutrons $\left(E_{n} \approx 1.1 \mathrm{MeV}\right)$, and its absolute efficiency is $5.8 \times 10^{-5}$ for the same source at a source-to-detector distance of $1 \mathrm{~m}$. A typical count rate for a contact measurement on a Type $30 \mathrm{~A}$ cylinder containing $2200 \mathrm{~kg}$ of $3.1 \%$ enriched $\mathrm{UF}_{6}$ is about 3200 courits $/ \mathrm{m}$ with a background of 50 to 100 counts $/ \mathrm{m}$. 

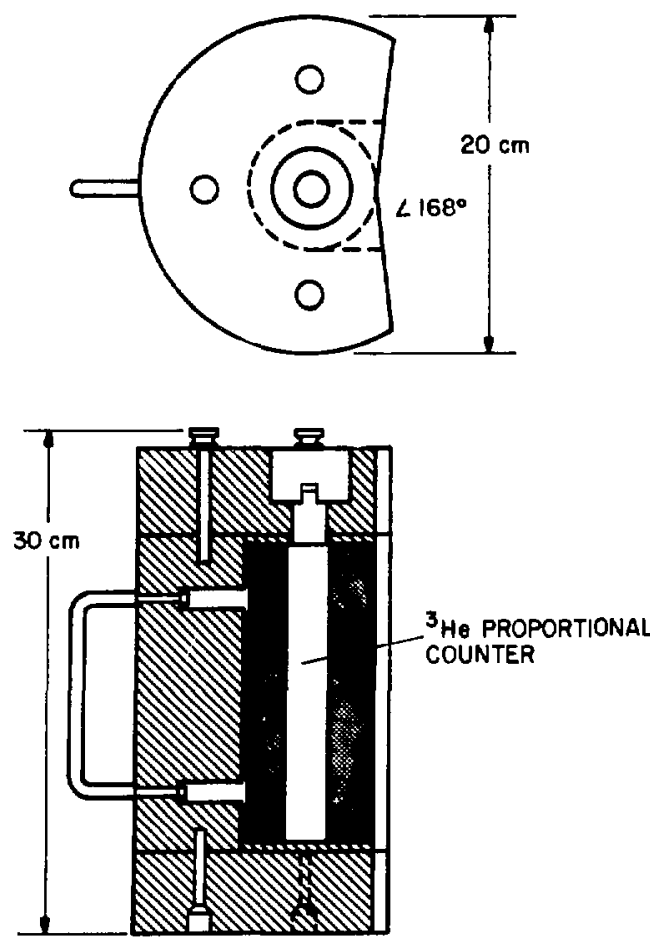

POLYETHYLENE

CADMIUM - WRAPPED POLYETHYLENE

Fig. 22.

${ }^{3}$ He neutron detector.

We used the SNAP detector with the Eberline SAM-II, described in Sec. IV-A, as well as the Eberline MS-2 Miniscaler. The complete verification system, consisting of SNAP detector, battery-powered electronics, cables, and batteries, is contained in one 61 - by $47-$ by $22-\mathrm{cm}$ suitcase, which is easily portable.

Field measurements ${ }^{5}$ coniirm the applicability of the constant $235 \mathrm{U} /{ }^{234} \mathrm{U}$ (Case 3) verification procedure. We made additional field verification measurements of $\mathrm{UF}_{6}$ cylinders with known ${ }^{234} \mathrm{U}$ values to check the stated mass and isotopic composition (Case 1) verification procedure over a range of ${ }^{234} \mathrm{U}$ content and cylinder fill heights. To pick an appropriate orientation for the measurements, we scanned several Type 30A cylinders. Figures 23 and 24 are circumferential scans of three 2-1/2-ton cylinders that have different fill heights. The inset in Fig. 23 shows the configuration. The shape of these scans agrees qualitatively with Forster and Walton's calculations; ${ }^{18}$ we positioned the detector at 4.5- and 7.5-h positions for our measurements. 


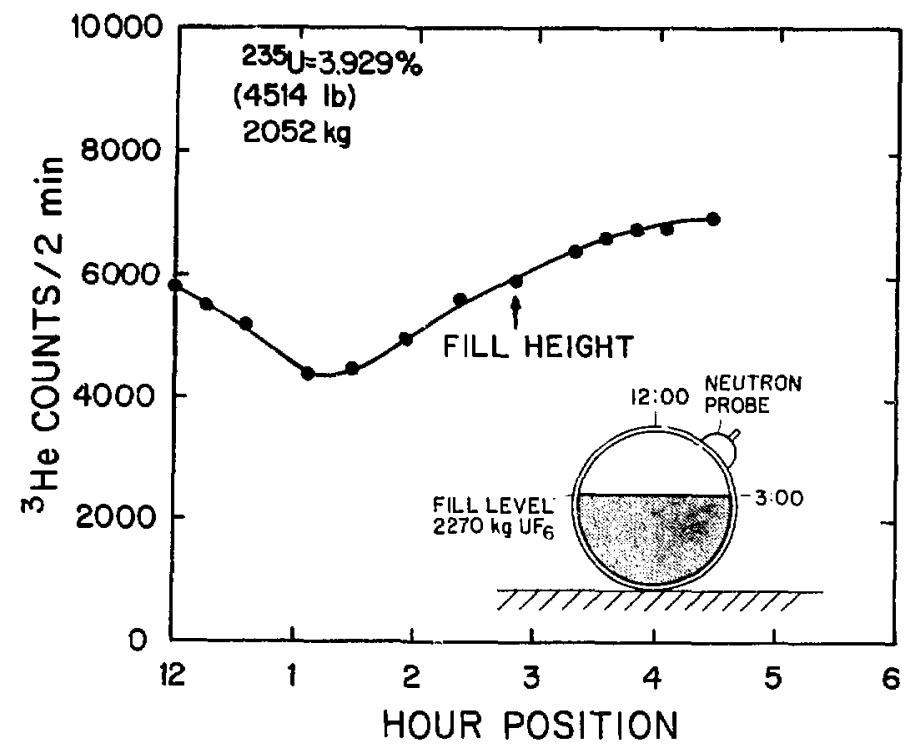

Fig. 23.

${ }^{3}$ He detector circumferential scan of a full Type $30 \mathrm{~A}$ cylinder.

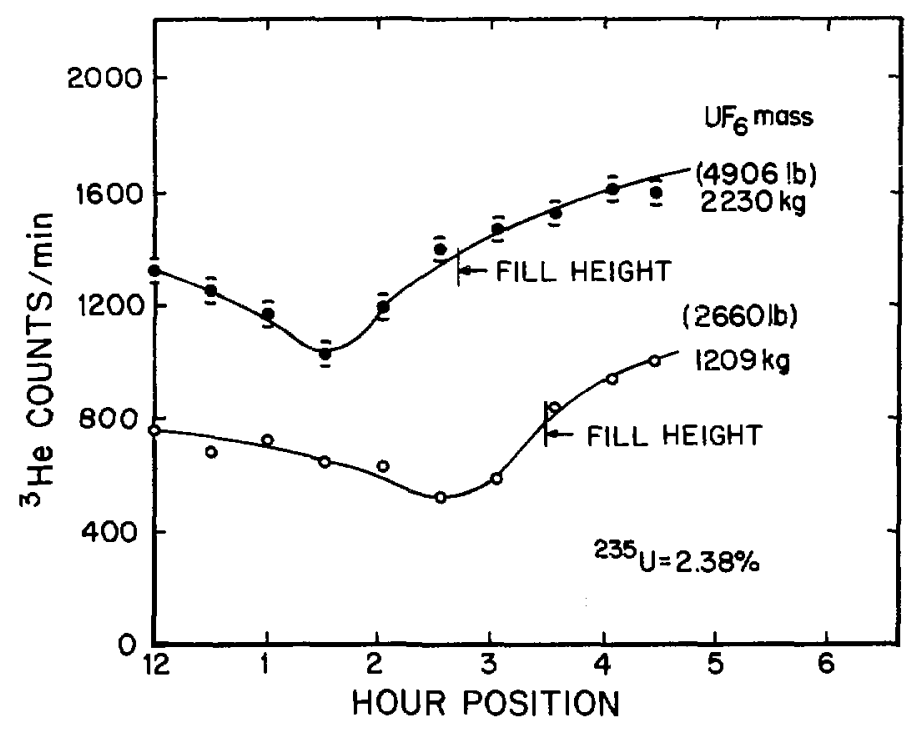

Fig. 24.

${ }^{3} \mathrm{He}$ detector circumferential scan of Type 30A cylinders of identical enrichment but different fill levels. 
Figure 25 shows the correlation between the measured ${ }^{3} \mathrm{He}$ neutron probe count rate and the calculated neutron source strength (Q) from Eq. (1), omitting the $236 \mathrm{U}$ term for 16 of the 18 Type $30 \mathrm{~A}$ cylinders that we examined. Data from two cylinders are not plotted: one had an apparent discrepancy in the stated ${ }^{234} \mathrm{U}$ content and the other had an extremely low fill mass of only $346 \mathrm{~kg}$. The UF 6 mass for the 16 cylinders ranged from 1000 to $2230 \mathrm{~kg}$ and $235 \mathrm{U} / 234 \mathrm{U}$ ratios ranged from 112 to 213 . The agreement between the measured count rate and $Q$ is quite linear, using mass and isotopic (Case 1) information.

We also analyzed the same data using the fixed ratio $235 \mathrm{U} / 234 \mathrm{U}$ (Case 3) information shown in Fig. 26. The count rate per unit mass and $235 \mathrm{U}$ enrichment are related linearly by $\mathrm{Eq}$. (7) $\left(\mathrm{Q} / \mathrm{M}_{\mathrm{U}} \simeq \mathrm{a}+\mathrm{bf}_{35}\right)$. The points in Fig. 26 exhibit scatter about the linear fit to the data because of variation in the $235 \mathrm{U} /{ }^{234} \mathrm{U}$ ratio, which the analysis assumes is constant.

\section{Passive Neutron Measurements with ${ }^{4}$ He Detectors}

An alternative approach, using a ${ }^{4} \mathrm{He}$ fast neutron detector for measuring the enrichment of large $\mathrm{UF}_{6}$ cylinders, samples a somewhat harder neutron spectrum. We investigated this approach for use in cylinder verification. The ${ }^{4} \mathrm{He}$ detector responds to fast neutrons, essentially those greater than $0.5 \mathrm{MeV}$.

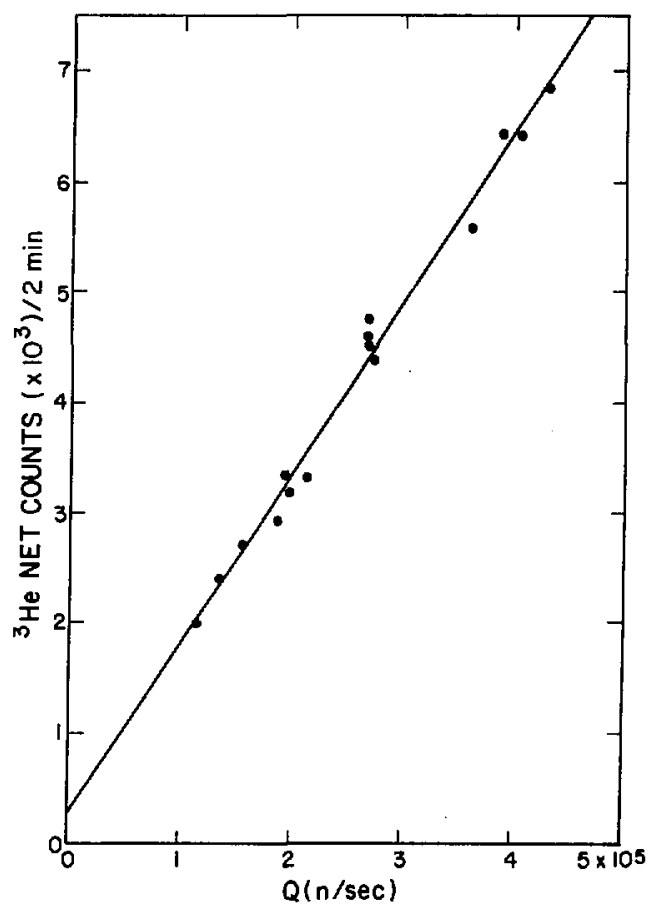

Fig. 25.

Measured neutron count rate compared to the neutron source strength calculated in Case 1. 


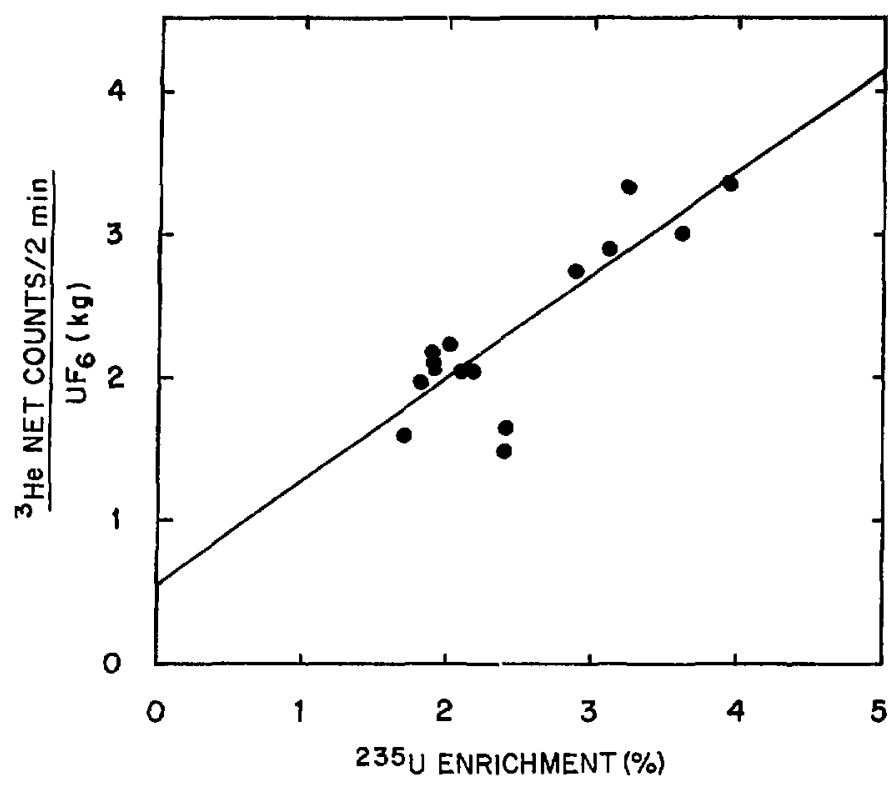

Fig. 26.

Measured neutron count rate per unit mass using Case 3 information.

The detector's peak response is to neutrons of about $1.1 \mathrm{MeV}$, with a gradual decrease in efficiency above that energy and a sharp decrease for neutrons of lower energy. Both $F(\alpha, n)$ and $238 \mathrm{U}$ spontaneous fission neutrons have average energies near $\perp \mathrm{MeV}$, hence the detector is well suited to counting neutrons from large UF 6 cylinders.

A theoretical prediction of expected neutron count clependence on cylinder mass and isotopic content was not available; therefore, we tried an empirical approach using net ${ }^{4} \mathrm{He}$ count rate data for 14 Type $30 \mathrm{~A}$ cylinders of nominal $2200-\mathrm{kg}$ content. Of these, two were filled to about: $1000 \mathrm{~kg}$, two filled to about $1800 \mathrm{~kg}$, and the remainder filled to a maximum of $2230 \mathrm{~kg}$. In addition to a wide mass range, the cylinders covered a $234 \mathrm{U}$ range of 0.0112 to $0.0307 \%$ and $235 \mathrm{U}$ enrichrinent range of 1.35 or $3.93 \%$. The cylinders were all the same length, diameter, and nominal wall thickness.

Our detector consisted of two $7.62-\mathrm{cm}$-diam by $30.48-\mathrm{cm}-$ long ${ }^{4} \mathrm{He}$ proportional counters at $18-\mathrm{atm}$ fill pressure. The tubes were mounted parallel to each other in a rigid framework with a 10-cm spacing between tubes. A $10-\mathrm{cm}-0 . d$. by $15-\mathrm{cm}-$ long polyethylene cylinder was mounted in the framework between tubes and served as a neutron source moderator for the ${ }^{4} \mathrm{He}$ active technique (described in Sec. IV-E). Even though the cylinder was empty, it remained in place during these passive measurements. The detector was placed in contact with $\mathrm{a} \mathrm{UF}_{6}$ cylinder at the midpoint of its length as close to the ground as possible. Measurements usually were taken on both sides of the cylinder and their average was used in the data analysis. 
The equation chosen to fit the data was of the form

$Y=M^{i}\left(A_{i}+B_{i} f_{34}\right)$,

where $\mathrm{Y}$ is the net observed count rate and $f_{34}$ is the fractional isotopic composition of ${ }^{234} \mathrm{U}$. We varied $\mathrm{i}$ and chose the value having the best correlation coefficient ( 1 represents perfect fit). The results for the correlation coefficient were: for $i=0$ (that is, $Y_{0}=A_{0}+B_{0} f_{34}$ ), a value of 0.08809 was obtained; for $i=1,0.8267$; and for the case $i=1 / 2$, a correlation coefficient of 0.9864 was obtained. Thus the $M^{1 / 2}$ correlation is quite good; the data points and fitted curve are shown in Fig. 27. Physical reasons for the observed $M^{1 / 2}$ dependence are not obvious; thus, it is possible that a different size detector or one used in a different detector-cylinder geometry could yield another result.

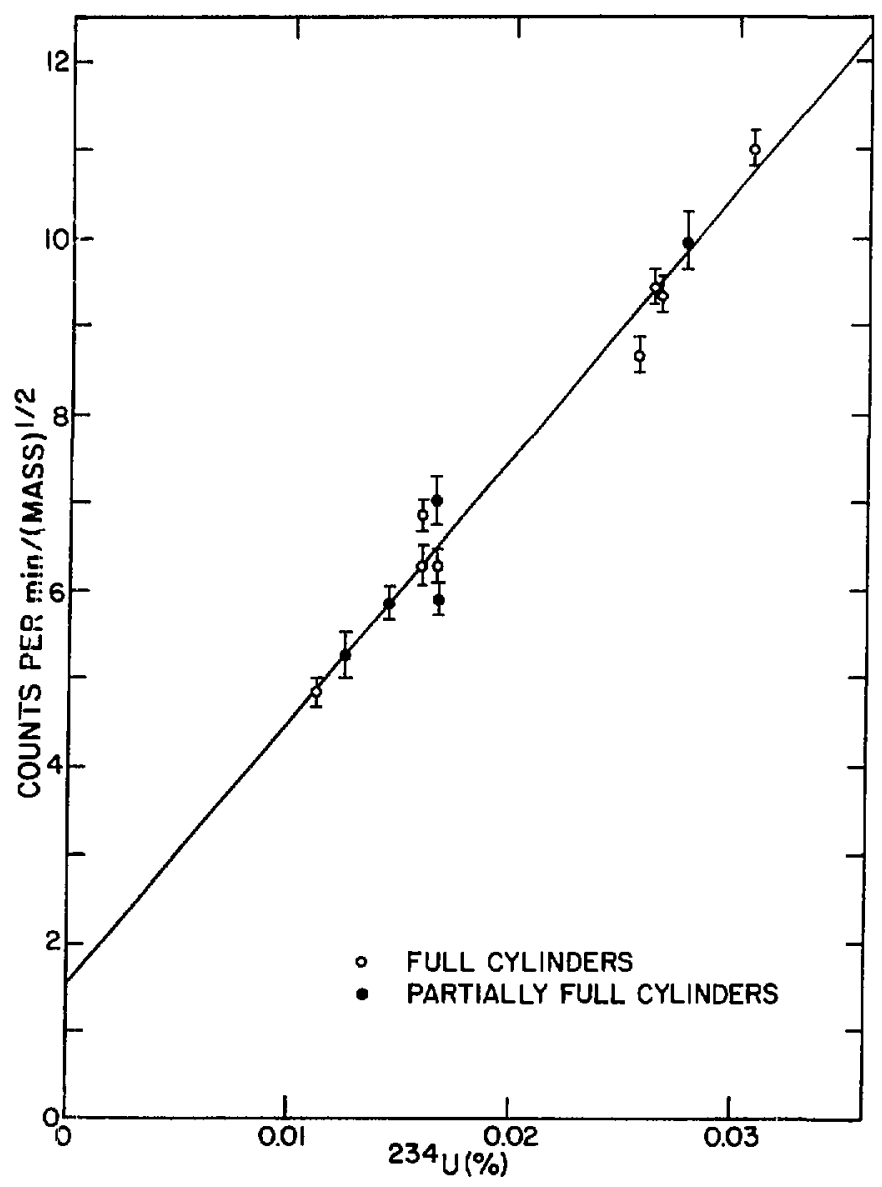

Fig. 27.

Passive ${ }^{4} \mathrm{He}$ detector results. 


\section{E. Active Neutron Interrogation and 4 He Detectors}

We developed an active neutron interrogation technique to verify the enrichment of $\mathrm{UF}_{6}$ in large cylinders. The technique uses the same ${ }^{4} \mathrm{He}$ detector described in Sec. IV-D for passive ${ }^{4} \mathrm{He}$ measurements and is valid for low-enriched material (less than $4 \%$ 235U). Caldwell gives a detailed description of the detectors and early field trials 20,21 and Walton describes a simplification to the technique and additional field trials. 22 Here we will briefly describe the technique and give measurement results for a varied group of large cylinders.

The detector being used for a small (Type 5A) cylinder verification in Fig. 28 contains a moderated $\operatorname{Li}(\alpha, \mathrm{n})$ neutron source $\left(1\right.$ to $\left.5 \times 10^{5} \mathrm{n} / \mathrm{sec}\right)$ of interrogating thermalized neutrons. We used both $238 \mathrm{Pu}-\mathrm{Li}$ and $24 \mathrm{I}_{\mathrm{Am}-\mathrm{Li}}$ sources; there is little difference between the two sources, but the latter is slightly preferable. Two measurements are required: one with the detector as shown and the other with a $0.3-\mathrm{mm}$-thick cadmium sheet between the detector and cylinder. The presence of the cadmium sheet removes the interrogating neutrons and gives a measure of natural and source-induced background. The difference between the two measurements (bare - cadmium) is a measure of the net induced fission in the $\mathrm{UF}_{6}$ cylinder and is directly related to the $235 \mathrm{U}$ content.

Data in Fig. 29 are measurements made of cylinders that were full or partly full. The $\mathrm{UF}_{6}$ contained in the cylinders varied from 1.35 to $3.93 \%$ $235 \mathrm{U}$, according to mass spectrographic analysis. The plotted count rate difference $Y$ was fitted with both linear (IY) and quadratic (2Y) functions of enrichment, the latter being a slightly better fit (correlation coefficient 0.96 versus 0.94 ), possibly because saturation of the measurement takes place with increasing enrichment. The average error here between points and curves is

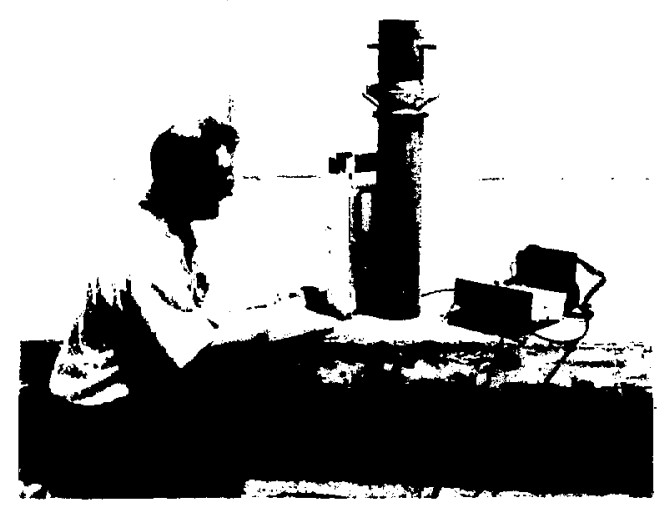

Fig. 28.

Active neutron interrogation of

a Type $5 A$ container of UF6. 
about $10 \%$ and in other measurements has been as good as $5 \%$. A lower limit for the fill mass was not precisely determined. Here half-full cylinders lie near the curves, but the one filled to about $15 \%$ is an outlier well below the curve.

The active neutron interrogation technique requires that a calibration curve, like one of those in Fig. 29, be established using known mass (or fill height) and enrichment $U_{6}$. Then other cylinders can be verified to perhaps $10 \%$ by making measurements using the same detector, interrogating source $(238 \mathrm{Pu}-\mathrm{Li}$ or $241 \mathrm{Am}-\mathrm{Li})$, and measurement position on the cylinder that was used in calibration. The technique can be used for $\mathrm{UF}_{6}$ or other forms of uranium (metals, for example) and is independent of the $234 \mathrm{U} / 235 \mathrm{U}$ ratio because it directly determines the amount of thermally fissioning isotope $235 \mathrm{U}$.

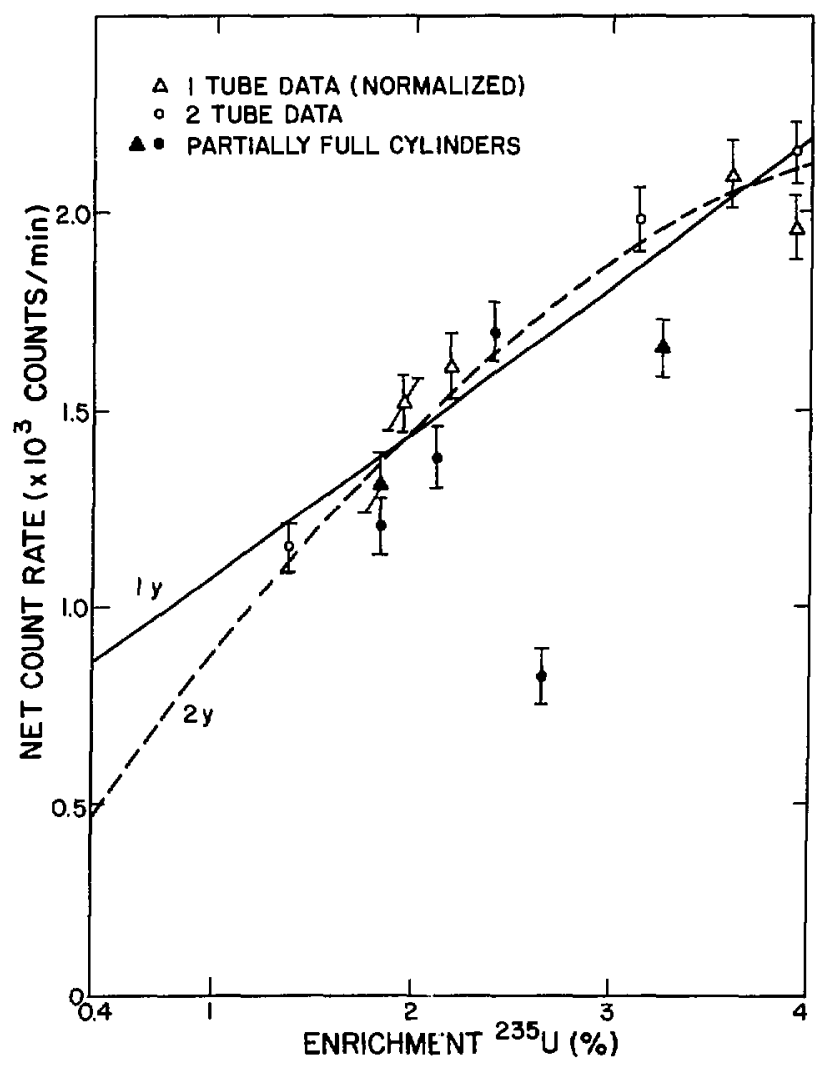

Fig. 29.'

Neutron interrogation response using a $\operatorname{Li}(\alpha, n)$ source and $4 \mathrm{He}$ detector. 
Radiography supplemented the information obtained by the foregoing techniques for verification of $\mathrm{UF}_{6}$ cylinders. A radiograph showing the distribution of $\mathrm{UF}_{6}$ in cylinders helped interpret the portable instrument results.

The radiography equipment we used consisted of a $200-\mathrm{Ci} 60 \mathrm{Co}$ gammaray source, radiographic paper type film $(35.6 \mathrm{~cm}$ by $43.2 \mathrm{~cm})$ with Kodak Industrex screens, and a paper film processor. The experimental arrangement in Fig. 30 used paper film with intensifying screen in light-tight cassettes in multiple cassette arrays for large cylinders. Radiographic exposure times and distances are given in Table IX for each cylinder type.

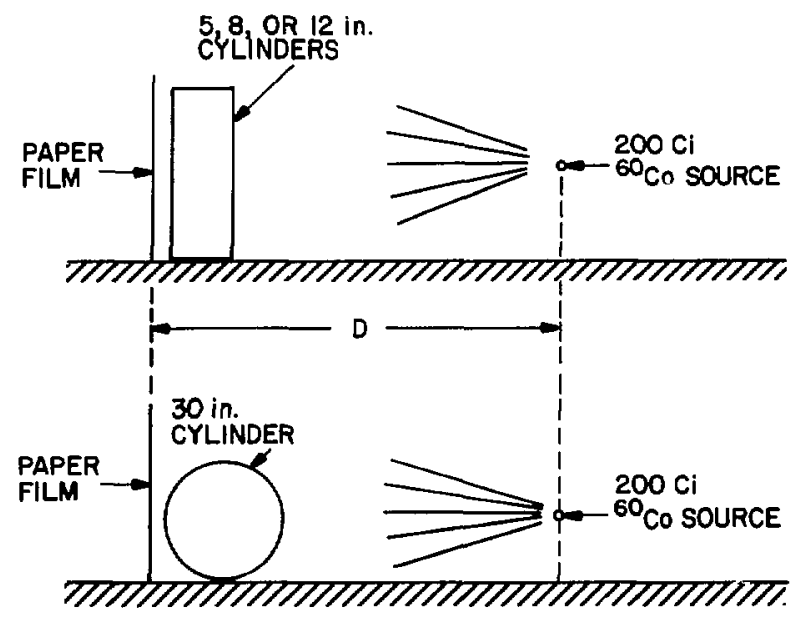

Fig. 30.

Geometry used for cylinder radiography.

TABLE IX

CYLINDER RADIOGRAPHIC PARAMETERS

Cylinder Type

$5 A$

$8 \mathrm{~A}$

$12 A$

$30 \mathrm{~A}$
Source-to-Screen

Separation $D(m)^{a}$

1.22

1.83

1.83

1.83
Exposure Time (min)

\section{2}

6

6

6-9

$\overline{\mathrm{a}}$ The parameter $\mathrm{D}$ appears in Fig. 30. 
Figures 31 to 34 are radiographs of Types $5 \mathrm{~A}, 8 \mathrm{~A}, 12 \mathrm{~A}$, and $30 \mathrm{~A}$ cylinders, respectively. Pairs of simultaneous radiographs made with different screens were used to span the variable depth of the UF $_{6}$. Radiographs show construction of the cylinders, the location and shape of the fill tube, the distribution of the $\mathrm{UF}_{6}$, and the presence of voids and density irregularities in the material. Comparing radiographs of many cylinders shows that the distribution of material varies substantially and appears to depend on the orientation, length, and shape of the fill tube.

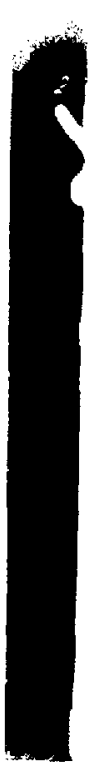

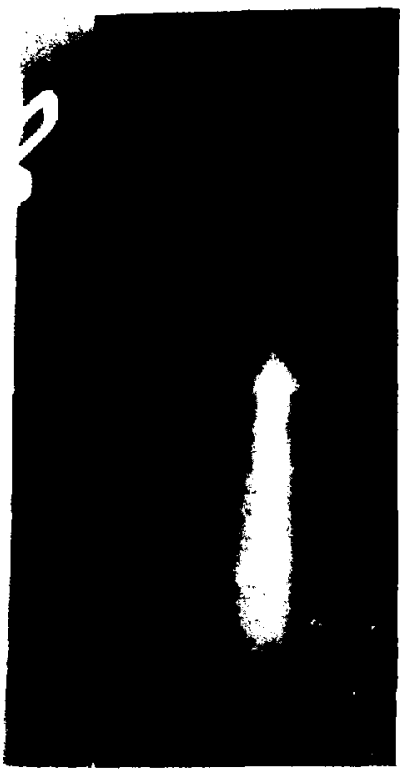

Fig. 31.

Type $5 \mathrm{~A} \mathrm{UF}_{6}$ cylinder radiograph.

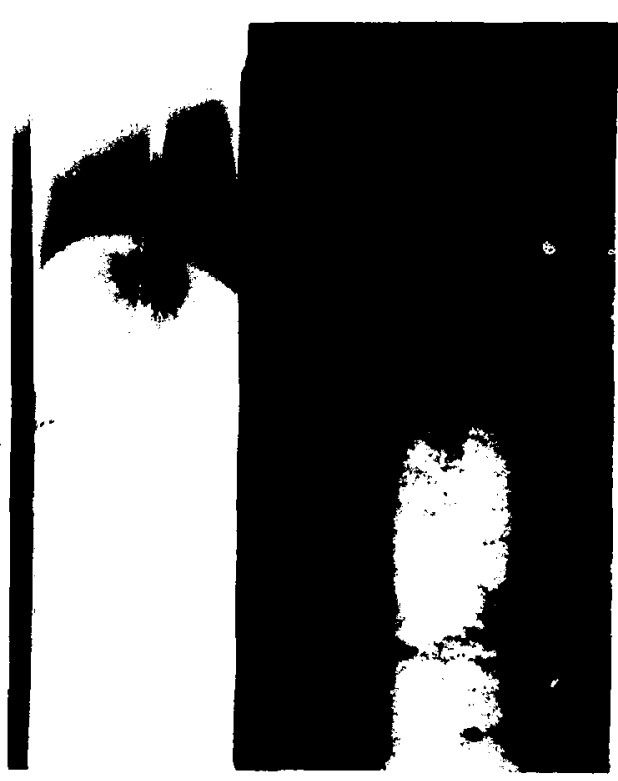

Fig. 32.

Type $8 \mathrm{~A}^{\mathrm{U}} \mathrm{UF}_{6}$ cylinder

radiograph. 


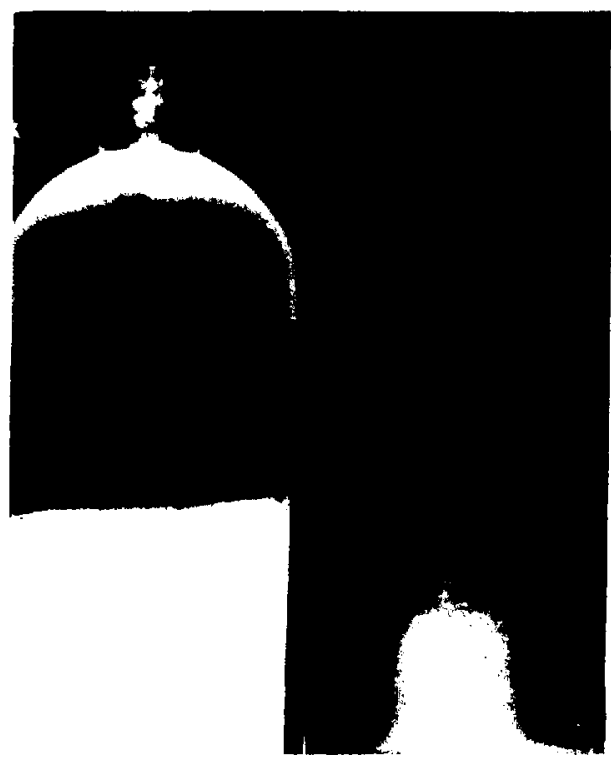

Fig. 33.

Type $12 \mathrm{~A} \mathrm{UF}_{6}$ cylinder radiograph.

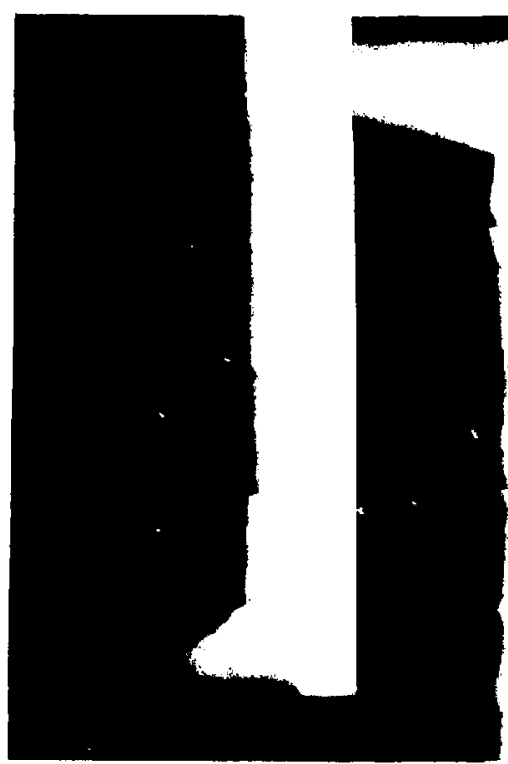

Fig. 34.

Type $30 \mathrm{~A} \mathrm{UF}_{6}$ cylinder radiograph.

\section{G. Summary of the Verification Techniques}

The verification techniques described in this section include gammaray and active and passive neutron measurements. The gamma-ray techniques are similar; both require homogeneity of the $\mathrm{UF}_{6}$ and are affected by voids or cracks in the $\mathrm{UF}_{6}$, by contaminant deposits, and by variation in wall thickness. The alternative gamma-ray method has some advantages but is subject to the additional time variation of $238_{\mathrm{U}} \mathrm{U}$ daughter activity. Both techniques sample a fairly shallow layer of $\mathrm{UF}_{6}$.

Neutron techniques sample a deeper region but also have drawbacks. The passive neutron measurements require knowledge of the ${ }^{234} \mathrm{U} /{ }^{235} \mathrm{U}$ isotope ratio and need a minimum fill height. The active technique depends on the ${ }^{235} \mathrm{U}$ content alone, but because it is an active technique, it incurs the occasional inconvenience associated with transporting a radioactive source.

We provided equipment to URENCO* to carry out the single gammaray enrichment technique and the two passive and one active neutron measurements. The URENCO trials are reported by Weller, 23 who was unable

\footnotetext{
*URENCO Limited, 40 West Street Marlow, Buckinghamshire, England.
} 
to use the ${ }^{4} \mathrm{He}$ techniques because a moist environment caused high-voltage breakdown in the detector wiring. The URENCO experience using the single gamma-ray and neutron techniques was much the same as ours except that URENCO heated containers to homogenize the cylinders and found differences over a period of a few months that made calibration, using samples of similar age, essential before product cylinders could be verifi $\_$d.

\section{v. CONCLUSIONS}

Some specific conclusions and recommendations emerge from our experience accumulated in this program.

- It is possible to fabricate portable radiation equipment that is adequate for verifying the isotopic content of $U F_{6}$ cylinders over the entire range of sizes and enrichments normally encountered. However, improvements to existing prototype equipment and commerical components are needed to improve performance and ease of use. Procedures to improve the accuracy and reproducibility of field measurements should also be refined. With improvements, measurements using the portable equipment together with information for enrichment plant design may be sufficient to verify material balances when applied continuously from plant startup.

- It is possible to fabricate personnel doorway monitors for unattended use that are adequate for small quantities of unshielded uranium. Improvements in detecting shielded uranium would be desirable in a final installation. Data storage and tamper-indicating techniques are satisfactory, but unnecessarily restrictive.

- Unattended shipping dock monitors are reliable ano sensitive enough to detect the large quantities of material implicit in undeclared feed. A conflict exists between this capability and that of personnel doorways in detecting enriched product, which may indicate that personnel monitors are superfluous in this situation. The conflict may be resolved for a specific plant.

- A design for a package monitor is highly dependent on specific plant models. An unattended system is probably not feasible. Therefore, administrative procedures for scheduling movements and negotiated agreements to control package configuration would be necessary. In view of the high potential for simple monitors to determine design information, further development and evaluation in a real centrifuge facility is necessary.

- Waste disposal monitoring is also highly specific and has not been addressed in this report.

- The surveillance provided by personnel, package, or shipping dock radiation monitors is effective only if additional means are employed to ensure that no undeclared routes exist for material flow.

- Isolated components of a safeguards system for enrichment plants have been developed and evaluated separately in this report. Effectiveness of the complete system can be determined by application to a facility in conjunction with appropriate nonnuclear techniques. 


\section{APPENDIX A}

\section{STANDARD UF 6 CYLINDER DATA}

All of the samples used in collecting data for this report were in standard-size containers that the US uranium enrichment industry regularly uses for $\mathrm{UF}_{6}$. A summary of information about container size and construction, extracted from Ref. 3, appears in Table A-l. To read the information in the table, scan each row across the two-page spread. 
TABLE A-1

\section{STANDARD UF 6 CYLINDER DATA}

\begin{tabular}{|c|c|c|c|c|}
\hline $\begin{array}{l}\text { Cylinder } \\
\text { Model } \\
\text { Number }\end{array}$ & $\begin{array}{c}\text { Nominal } \\
\text { Diameter } \\
\text { (in.) }\end{array}$ & $\begin{array}{l}\text { Wall } \\
\text { Thickness } \\
\text { (in.) }\end{array}$ & $\begin{array}{c}\text { Construction } \\
\text { Material }^{\mathrm{a}} \\
\end{array}$ & $\begin{array}{l}\text { Minimum } \\
\text { Volume } \\
\text { (cu ft) }\end{array}$ \\
\hline IS & 1.5 & 0.063 & nickel & 0.0053 \\
\hline $2 S$ & 3.5 & 0.112 & nickel & 0.0254 \\
\hline $5 A$ & 5 & 0.250 & Monel & 0.284 \\
\hline $8 \mathrm{~A}$ & 8 & 0.188 & Monel & 1.319 \\
\hline $12 \mathrm{~A}$ & 12 & 0.200 & nickel & 2.38 \\
\hline $12 \mathrm{~B}$ & 12 & 0.200 & Monel & 2.38 \\
\hline $30 \mathrm{~A}$ & 30 & 0.406 & steel & 25.65 \\
\hline $30 B^{d}$ & 30 & 0.500 & steel & 26.0 \\
\hline $48 \mathrm{~A}$ & 48 & 0.625 & steel & 108.9 \\
\hline $48 x^{f}$ & 48 & 0.625 & steel & 108.9 \\
\hline $48 \mathrm{~F}$ & 48 & 0.625 & steel & 140.0 \\
\hline $48 Y^{f}$ & 48 & 0.625 & steel & 142.7 \\
\hline
\end{tabular}

a For packaging normal and depleted $\mathrm{UF}_{6}$, cylinders of other materials such as steel and Monel may be substituted, provided they have equivalent strength.

$\mathrm{b}_{\mathrm{Fill}}$ limits are based on $250^{\circ} \mathrm{F}$ maximum $\mathrm{UF}_{6}$ temperature $(203.3 \mathrm{lb}$ $\mathrm{UF}_{6} / \mathrm{cu} \mathrm{ft}$ ), certified minimum internal volumes for all cylinders, and a minimum safety factor of $5 \%$. The operating limits apply to $\mathrm{UF}_{6}$ with a minimum purity of $99.5 \%$. More restrictive measures are required if additional impurities are present. The maximum $\mathrm{UF}_{6}$ temperature must not be exceeded.

cMaximum enrichments indicated require moderation control equivalent to a $\mathrm{UF}_{6}$ purity of $99.5 \%$. Without moderation control, the maximum permissible enrichment is $1.0 \mathrm{wt} \% 238_{\mathrm{U}}$. 
TABLE A-I (continued)

\begin{tabular}{|c|c|c|c|c|}
\hline \multirow[b]{2}{*}{$\begin{array}{l}\text { Nomina! } \\
\text { Length } \\
\text { (in.) }\end{array}$} & \multirow{2}{*}{$\begin{array}{l}\text { Approximate } \\
\text { Tare Weight } \\
\text { (without valve } \\
\text { protector) (lb) }\end{array}$} & \multirow[b]{2}{*}{$\begin{array}{l}\text { Maximum } \\
\text { Enrichment } \\
\text { wt } \% 238 \mathrm{U} \\
\end{array}$} & \multicolumn{2}{|c|}{ Fill Limit } \\
\hline & & & $\begin{array}{l}\text { Maximumb } \\
\mathrm{lb} \mathrm{UF}_{6} \\
\end{array}$ & $\begin{array}{l}\text { Minimum } \\
{\mathrm{lb} U \mathrm{~F}_{6}}\end{array}$ \\
\hline 11.0 & 1.75 & 100.0 & 1.0 & 0.1 \\
\hline 11.5 & 4.2 & 100.0 & 4.9 & 1 \\
\hline 36 & 55 & 100.0 & 55 & 11 \\
\hline 56 & 120 & 12.5 & 255 & 55 \\
\hline 54 & 185 & 5.0 & 460 & 55 \\
\hline 54 & 185 & 5.0 & 460 & 55 \\
\hline 81 & 1400 & $5.0^{C}$ & 4950 & $2300^{\mathrm{e}}$ \\
\hline 81 & 1400 & $5.0^{\mathrm{C}}$ & 5020 & $2300^{\mathrm{e}}$ \\
\hline-- & 4500 & $4.5^{c}$ & 21030 & 14000 \\
\hline 121 & 4500 & $4.5^{c}$ & 21030 & 14000 \\
\hline-- & 5200 & $4.5^{c}$ & 27030 & 21000 \\
\hline 150 & 5200 & $4.5^{c}$ & 27560 & 21000 \\
\hline
\end{tabular}

${ }^{\mathrm{d}}$ This cylinder replaces the Type 30A cylinder.

eShipments from the Energy Research and Development Administration (ERDA) and at the customer's request can be less than $2300 \mathrm{lb}$ in the Type $30 \mathrm{~A}$ or $30 \mathrm{~B}$ cylinders, providing the $\mathrm{UF}_{6}$ is transferred from a larger cylinder.

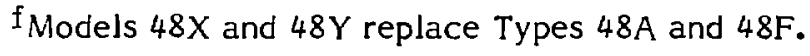


$-50$ 


\section{APPENDIX B}

\section{CALCULATED RESPONSE FOR A}

TWO-GAMMA-RAY ENRICHMENT METER

Consider a sample of $\mathrm{UF}_{6}$ inside a cylinder. The number of gamma rays detected by a detector after attenuation in the container wall is given by

$$
C=\varepsilon C^{\prime} e^{-\mu} c^{x}
$$

where $C^{\prime} \quad=\quad$ count for a bare sample without the container,

$\varepsilon \quad=\quad$ detector efficiency, a function of gamma-ray energy and the solid angle seen by the detector,

$\mu_{c} \quad=$ attenuation coefficient of the container wall,

$\mathrm{x} \quad=$ container. wall thickness, and

C $=$ count recorded for the sample in the container.

The attenuation of gamma rays from self-absorption in the sample reduces the count $C^{\prime}$, as indicated by the expression

$$
C^{\prime}=C_{0} \frac{1}{\mu d}\left(1-e^{-\mu d}\right),
$$

where $\mu=$ attenuation coefficient of the sample,

d $=$ sample thickness, and

$\mathrm{C}_{0} \quad=$ total number of gammas produced in the sample.

It should be noted that

$C_{0}=f N$,

where, for a given gamma ray, $f$ is the specific intensity in $\gamma / \mathrm{sec} \cdot g$ and $N$ is the mass of material in grams. Combining Eqs. (BI), (B2), and (B3) and solving for $\mathbf{N}$ gives

$$
N=C \frac{\mu d}{\varepsilon f} \cdot \frac{e^{\mu_{c} x}}{1-e^{-\mu d}}
$$


Equations (B1) and (B2) assume good geometry can be maintained in some poor situations, such as a short source-to-detector distance, by collimating the detector.

Using Eq. (B4), the ratio $\mathrm{N}_{238} / \mathrm{N}_{235}$ is the ratio of the number of $238 \mathrm{U}$ nuclei to the ${ }^{235} \mathrm{U}$ nuclei and is determined by the $1001-\mathrm{keV}$ and $186-\mathrm{keV}$ gamma rays as

$\frac{N_{238}}{N_{235}}=\frac{C_{1001}}{C_{186}} \cdot \frac{f_{186}}{f_{1001}} \cdot \frac{\varepsilon_{186}}{\varepsilon_{1001}} \cdot \frac{\mu_{1001}}{\mu_{186}} e^{\left(\mu_{c 1001}-\mu_{c 186}\right)_{x}} \cdot \frac{1-e^{-\mu_{186} d}}{1-e^{-\mu_{1001}}} \cdot$

Another equation obtained, using the 766-keV gamma ray instead of the 100.1-keV gamma ray, could be used. If minor isotope contributions in the sample are negligible, Eq. (B5) is related to enrichment by

$$
\%^{235} \text { U enrichment }=\frac{100}{1+N_{238} / N_{235}}
$$

We calculated Eq. (B5) for Type $5 A$ cylinders of $U_{6}$ using parameters for a $4.45-\mathrm{cm}$ by $5.08-\mathrm{cm} \mathrm{NaI(T \ell )}$ detector and for Types 1S, 5A, 12A, 30A, and $48 \mathrm{~A}$ cylinders of $\mathrm{UF}_{6}$, using parameters for a $\mathrm{Ge}(\mathrm{Li})$ detector. The cylinders are made either of nickel or of iron, and the appropriate attenuation coefficients ${ }^{24}$ for nickel, iron, and $\mathrm{UF}_{6}$ are listed in Table B-I.

The values of $f_{186}$ and $f_{1001}$ were obtained from the decay rate of $235 \mathrm{U}$ and $238 \mathrm{U}$ converted to a specific decay rate for $\mathrm{UF}_{6}$. The number of $186-\mathrm{keV}$ gamma rays per decay of the $235 \mathrm{U}$ nucleus 25 and the number of $1001-\mathrm{keV}$ gamma rays per decay of the ${ }^{238} \mathrm{U}$ nucleus 26 were used to obtain a specific gamma emission rate in $\mathrm{\gamma} / \mathrm{sec} \cdot \mathrm{g} \mathrm{UF}_{6}$ for ${ }^{235} \mathrm{U}$ and $238_{\mathrm{U}}$. For the NaI(Tl) detector, gamma rays with energies near the $186-\mathrm{keV}$ and $1001-\mathrm{keV}$ lines were also included in determining $\mathrm{f}_{186}$ and $\mathrm{f}_{1001}$. Table B-II gives the values used in Eq. (B5) for the calculations relating counts to enrichment for Types IS and 5 A cylinders.

\section{TABLE B-I}

\section{ATTENUATION COEFFICIENTS USED IN CALCULATIONS}

\begin{tabular}{lll}
$\begin{array}{c}\text { Attenuation } \\
\text { Coefficient }\end{array}$ & $\begin{array}{l}\text { Coefficient } \\
\text { Value }\left(\mathrm{cm}^{-1}\right)\end{array}$ & Material \\
\cline { 2 - 3 } & 5.19 & $\mathrm{UF}_{6}$ \\
$\mu_{186}$ & 0.359 & $\mathrm{UF}_{6}$ \\
$\mu_{1001}$ & 1.37 & nickel \\
$\mu_{\mathrm{cl}} 86$ & 0.534 & nickel \\
$\mu_{\mathrm{cl}} 001$ & 1.10 & iron \\
$\mu_{\mathrm{cl}}$ & 0.46 & iron \\
$\mu_{\mathrm{cl} 1001}$ & &
\end{tabular}


TABLE B-II

VALUES OF PARAMETERS AND FACTORS USED IN CALCULATIONS

Parameter

\begin{tabular}{|c|c|c|c|c|c|c|}
\hline $\begin{array}{l}\text { Container } \\
\text { and Detector }\end{array}$ & $\begin{array}{c}f_{186} \text { of }{ }^{235} \mathrm{UF}_{6} \\
(\mathrm{r} / \mathrm{sec} \cdot \mathrm{g}) \\
\end{array}$ & $\begin{array}{c}f_{1001} \text { of }{ }^{238}{ }^{23 / \mathrm{sec} \cdot g)} \\
\end{array}$ & $x(\mathrm{~cm})$ & $d(\mathrm{~cm})$ & $\varepsilon_{186}$ & $\varepsilon_{1001}$ \\
\hline $\begin{array}{l}\text { Type 5A } \\
\text { Cylinders } \\
\text { and NaI(TR) } \\
\text { Detectors }\end{array}$ & $3.53 \times 10^{4}$ & 51.41 & 0.653 & 11.4 & 0.216 & 0.0288 \\
\hline $\begin{array}{l}\text { Type IS } \\
\text { Cylinders } \\
\text { and Ge(Li) } \\
\text { Detectors }\end{array}$ & $2.929 \times 10^{4}$ & 50.46 & 0.159 & 3.49 & 0.00430 & 0.00604 \\
\hline
\end{tabular}

The results in Fig. 21 for Type 5A cylinders of UF 6 and a NaI(Tl) detector may be used to determine percent enrichment by measuring $\mathrm{C}_{186} / \mathrm{C}_{1001}$ for a Type $5 \mathrm{~A}$ cylinder using a $4.45-\mathrm{cm}$ by $5.08-\mathrm{cm}$ NaI(Tl) detector and reading the enrichment from the curve in the figure. Similar curves were plotted for the other cases. 


\section{REFERENCES}

1. G. A. Hammond and L. R. Stieff, "Development of a Safeguards System for Containment and Surveillance at Uranium Enrichment Plants," IAEA SM201/11, 1977.

2. Herbert Kouts, "Reference Uranium Enrichment Pler.c," Brookhaven National Laboratory, Technical Support Organization Report, December 6, 1972.

3. "Uranium Hexafluoride: Handling Procedures and Container Criteria," ORO-651, Rev. 3, U.S. Atomic Energy Commission (1972).

4. 1. Werle, "Spectrum Measurements of Radioactive Neutron Sources in the $10 \mathrm{keV}$ - $10 \mathrm{MeV}$ Energy Region with Proton-Recoil Counters," Karlsruhe Nuclear Research Center report INR-4170-25 (Feb. 1970), Translation OR NL-tr-2415.

5. R. B. Walton, T. D. Reilly, J. L. Parker, J. H. Menzel, E. D. Marshall, and L. W. Fields, "Measurements of $\mathrm{UF}_{6}$ Cylinders with Portable Instruments," Nuclear Technology, 21 133 (1974).

6. Thomas E. Sampson, "Neutron Yields from Uranium Isotopes in Uranium Hexafluoride," Nucl. Sci. Eng. 54470 (1974).

7. W. H. Chambers, H. F. Atwater, P. E. Fehlau, R. D. Hastings, C. N. Henry, W. E. Kunz, T. E. Sampson, T. H. Whittlesey, and G. M. Worth, "Portal Monitor for Diversion Safeguards," Los Alamos Scientific Laboratory report LA-5681 (1974).

8. W. H. Chambers and J. F. Ney, "Tamper-Indicating Radiation Surveillance Instrumentation", Safeguarding Nuclear Materials, Vol. Il (1976), pp. 297-304; IAEA Symposium, Vienna, IAEA SM 201/12, October 20-24, 1975.

9. P. E. Fehlau, T. E. Sampson, C. N. Henry, J. M. Bieri, and W. H. Chambers, "On-Site Inspection Procedures for SNM Doorway Monitors," Los Alamos Scientific Laboratory report LA-7646 (1979).

10. J. V. Boyd, "Sandia Laboratories Personnel Doorway Monitor, Assessment and Evaluation," AEE Winfrith, CSR(76)286 (1976).

11. Project Engineering Division 1759, Sandia National Laboratories, "Development of an Unattended Personnel Portal for International Safeguards," Sandia Laboratories report SAND-80-1196 (1980), pp. 60-64.

12. Theodore Rockwell, Reactor Shielding Design Manual, (D. Van Nostrand Co., Princeton, New Jersey, 1957).

13. W. E. Kunz, C. N. Henry, G. M. Worth, and R, D. Hastings, "Hand-Held Personnel and Vehicle Monitors," Nuclear Materials Management IV, No. III (1975), p. 246. 
14. W. E. Kunz, W. H. Chambers, C. N. Henry, S. W. France, D. R. Millegan, R. D. Hastings, and G. M. Worth, "Hand-Held Personnel and Vehicle Monitors," Los Alamos Scientific Laboratory report LA-6359 (1976).

15. G. W. Smith and L. G. Rice, III, "An Active Neutron Technique for Detecting Attempted SNM Diversion," in Measurement Technology for Safeguards and Material Control, NBS Special Publication 582 (1980), p. 372.

16. W. E. Kunz, J. D. Atencio, and J. T. Caldwell, "Pulsed-Neutron Classified Waste Monitor," in "Nuclear Safeguards Research and Development Program Status Report, January-March 1980," G. R. Keepin, Ed., Los Alamos Scientific Laboratory report LA-8373-PR (1980), pp. 28-29.

17. T. D. Reilly, R. B. Walton, and J. L. Parker, "The Enrichment Meter - A Simple Method for Measuring Isotopic Enrichment," in "Nuclear Safeguards Research and Development Program Status Report, September-December 1970," G. R. Keepin, Ed., Los Alamos Scientific Laboratory report LA-4605-MS (1970), PP. 19-21.

18. R. A. Forster and R. B. Walton, "Neutron Multiplication and Leakage Characteristics for UF 6 Cylinders," in "Nuclear Analysis Research and Development Program Status Report, September-December 1973," Los Alamos Scientific Laboratory report LA-5557-PR (1974), pp. 6-8.

19. R. B. Walton and T. L. Atwell, "A Portable Neutron Detector--SNAP," in "Nuclear Analysis Research and Development Program Status Report, January-April 1973," Los Alamos Scientific Laboratory report LA-529I-PR (1973), pp. 14-15.

20. J. T. Caldwell, "New Techniques for $235 \mathrm{U}$ Enrichment Determination in UF $_{6}$ Cylinders," Nucl. Materials Management, Vol. II, No. II (1973), D. 16.

21. J. T. Caldwell, J. L. Evans, P. E. Fehlau, and T. H. Whittlesy, "235U Enrichment Determination Using "He Proportional Counters," Proc. ANS Meeting, San Francisco, California, November 1973.

22. R. B. Walton, T. D. Reilly, J. L. Parker, J. H. Menzel, E. D. Marshall, and L. W. Fields, "Measurements of $\mathrm{UF}_{6}$ Cylinder with Portable Instruments," Nuclear Technology 21, 133 (1974).

23. P. J. Weller, "Report on the Tests of American Safeguards Equipment in the Tripartite Centrifuge Pilot Plants, October 1974-March 1975," URENCO Limited report UTR54 (April 1975).

24. E. Storm and H. I. Israel, "Photon Cross Sections from $1 \mathrm{keV}$ to $100 \mathrm{MeV}$ for Elements $Z=1$ to $Z=100$," Nuclear Data Tables A7, 565 (1970).

25. A. H. Jaffey, K. F. Flynn, L. E. Glendenin, W. C. Bentley, and A. M. Essling, "Precision Measurement of Half-Lives and Specific Activities of $235 \mathrm{U}$ and 238 U," Phys. Rev. C4, 1889 (1971).

26. C. Marsol and G. Ardisson, "Radioactivite. Etude du rayonnement $\gamma$ qui accompagne la desintegration de ${ }^{234 \mathrm{~m}} \mathrm{~Pa}$," Compt. Rend. B274, 429 (1972). 


\section{GLOSSARY}

$\begin{array}{ll}\text { ACDA } & \text { Arms Control anc Disarmament Agency } \\ \text { DOE } & \text { Department of Energy } \\ \text { DRM } & \text { digital rate multiplier } \\ \text { HEU } & \text { highly enriched uranium } \\ \text { IAEA } & \text { International Atomic Energy Agency } \\ \text { LLD } & \text { lower level discriminator } \\ \text { LWR } & \text { light-water reactor } \\ \text { NBS } & \text { National Bureau of Standards } \\ \text { SAM-II } & \text { Stabilized Assay Meter Il (Eberline Instrument Corp.) } \\ \text { SCA } & \text { single channel analyzer } \\ \text { SNAP } & \text { shielded neutron assay probe } \\ \text { SNM } & \text { special nuclear material } \\ \text { UK } & \text { United Kingdom } \\ \text { US } & \text { United States }\end{array}$

